ARTICLE

https://doi.org/10.1038/s41467-019-12983-z

Corrected: Author correction

\title{
Lineage tracing of acute myeloid leukemia reveals the impact of hypomethylating agents on chemoresistance selection
}

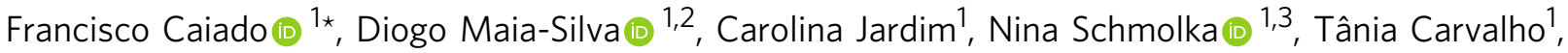 \\ Cláudia Reforço (1) ${ }^{1}$, Rita Faria (1) 1, Branka Kolundzija', André E. Simões' ${ }^{1}$ Tuncay Baubec (10 ${ }^{3}$, \\ Christopher R. Vakoc ${ }^{2}$, Maria Gomes da Silva ${ }^{4}$, Markus G. Manz (1) ${ }^{5}$, Ton N. Schumacher (10 ${ }^{6}$, \\ Håkan Norell ${ }^{1,7 \star} \&$ Bruno Silva-Santos (1) ${ }^{1,7 \star}$
}

Chemotherapy-resistant cancer recurrence is a major cause of mortality. In acute myeloid leukemia (AML), chemorefractory relapses result from the complex interplay between altered genetic, epigenetic and transcriptional states in leukemic cells. Here, we develop an experimental model system using in vitro lineage tracing coupled with exome, transcriptome and in vivo functional readouts to assess the AML population dynamics and associated molecular determinants underpinning chemoresistance development. We find that combining standard chemotherapeutic regimens with low doses of DNA methyltransferase inhibitors (DNMTi, hypomethylating drugs) prevents chemoresistant relapses. Mechanistically, DNMTi suppresses the outgrowth of a pre-determined set of chemoresistant AML clones with stemness properties, instead favoring the expansion of rarer and unfit chemosensitive clones. Importantly, we confirm the capacity of DNMTi combination to suppress stemnessdependent chemoresistance development in xenotransplantation models and primary AML patient samples. Together, these results support the potential of DNMTi combination treatment to circumvent the development of chemorefractory AML relapses.

\footnotetext{
${ }^{1}$ Instituto de Medicina Molecular João Lobo Antunes, Faculdade de Medicina, Universidade de Lisboa, Lisboa, Portugal. ${ }^{2}$ Cold Spring Harbor Laboratory, Cold Spring Harbor, New York, NY, USA. ${ }^{3}$ Department of Molecular Mechanisms of Disease, University of Zurich, Zurich, Switzerland. ${ }^{4}$ Instituto Portugues de Oncologia-Francisco Gentil, Lisbon, Portugal. ${ }^{5}$ Department of Medical Oncology and Hematology, University Hospital Zurich and University of Zurich, Zürich, Switzerland. ${ }^{6}$ Netherlands Cancer Institute, Amsterdam, The Netherlands. ${ }^{7}$ These authors jointly supervised this work: Håkan Norell, Bruno SilvaSantos. *email: caiado12@gmail.com; haakannorell@gmail.com; bssantos@medicina.ulisboa.pt
} 
C hemotherapy resistance (chemoresistance) is a major driver of cancer recurrence ${ }^{1}$. Intra-tumor heterogeneity (ITH), the end product of co-existing microenvironmental, phenotypical, transcriptomic, epigenetic, and genetic variants, drives chemoresistance by providing multiple substrates for tumor escape under the selective pressure of chemotherapy ${ }^{2}$. Genetic ITH results from continuous cycles of mutation, selection and expansion under context-specific selective pressures-i.e. cancer clonal evolution-and contributes to AML chemorefractory relapses through expansion of sub-clonal population(s) harboring either intrinsic (pre-existing) or acquired (therapy-induced) chemoresistance-promoting mutations ${ }^{1,3}$. In addition to genetic ITH, non-genetic ITH is also a major contributor to chemoresistance development. Heterogeneous epigenetic regulation of gene expression has been shown to generate hierarchally related but phenotypically divergent co-existing cell subpopulations originating from genetically identical tumor cells ${ }^{4}$. A clinically relevant example of non-genetic hierarchical organization of some tumors are cancer stem cells (CSC), which are genetically identical to the bulk of the tumor, but display substantially higher tumorigenic capacity than their isogenic siblings. CSCs have been implicated in chemoresistance and recurrence in various cancer types, which arise as a result of their unique properties. These are often encompassed in the term stemness, and include slow cell cycle progression (or quiescence), upregulation of drug-efflux pumps, protection from reactive oxygen species, high self-renewal or tumor initiation capacity in immunocompromised mice ${ }^{5}$.

Development of chemoresistant relapses is of particular importance in acute myeloid leukemia (AML), making this the deadliest blood cancer ${ }^{6}$. AML relapse to standard chemotherapy has been traced to pre-existing genetically defined clones that acquire additional mutations, evolving into the dominant relapsing sub-clones ${ }^{7-10}$. Despite the established role of specific genetic alterations in diagnosis, prognosis and treatment stratification $^{11-14}$, AML is a highly heterogeneous disease with surprisingly lower average number of mutations than most other adult cancers $^{15,16}$, suggesting that non-genetic factors are also relevant in AML outcomes. In fact, it was shown that AML relapse to standard chemotherapy depends heavily on transcriptional stemness programs ${ }^{17-19}$. The pervasiveness of non-genetic ITH is also evidenced by the existence of extensive epigenetic alterations in AML genomes. For example DNA methylation, an epigenetic modification that impacts transcription, carries diagnostic and prognostic value in $\mathrm{AML}^{20-22}$, with recent studies establishing DNA hypermethylation as a poor prognosis factor in de novo $\mathrm{AML}^{23}$. In contrast to genetic changes, epigenetic modifications are frequently reversible, which provides opportunities for their reversion to non-pathogenic states by the use of specific inhibitors. DNA methyltransferase inhibitors (DNMTi) or hypomethylating agents like decitabine (DAC) and azacitadine (Aza) have established clinical benefits in myelodysplastic syndrome (MDS) patients and are also approved as monotherapy for certain groups of elderly AML patients ${ }^{24}$. Importantly, different clinical studies combining low doses of DAC with standard chemotherapy regimens have shown clinical benefit, particularly in patients with refractory/relapsed $\mathrm{AML}^{25-27}$. In spite of its clinical benefit, the impact of combining DNMTis with chemotherapeutic regimens on the different layers of ITH and its overall effect on de novo AML chemoresistance development remains largely unexplored, mostly due to the lack of adequate experimental systems. A key approach to assess the impact of different therapies on ITH is the use of lineage-tracing technologies. Recent in vitro lineage-tracing studies have revealed the pre-existing nature of targeted therapy resistance in lung cancer and chronic myeloid leukemia models ${ }^{28}$, thus attesting the validity of in vitro lineage tracing experimental systems to dissect the consequences of ITH on cancer biology.
Here, we employ lineage tracing (DNA barcodes-BCs) to assess the longitudinal clonal dynamics, beyond the level of genetics, that underlie the emergence of chemoresistance in human AML (hAML) cells in the presence or absence of DNMTi. To assess the contribution of different layers of ITH, we combine lineage tracing with exomic, transcriptomic and phenotypic profiling together with in vivo functional assessment of hAML cells relapsing to chemotherapy. Using this approach, we find that standard chemotherapy drives the selection of a pre-determined set of recurring AML clones with increased in vivo leukemia-initiating capacity and resistance to a second round of chemotherapy. Strikingly, we reveal that low-dose DNMTi in combination with chemotherapy selects for an alternative unpredictable set of clones with decreased stemness properties that remain sensitive to chemotherapy. Collectively, our findings attest the potential of a combinatorial approach of standard chemotherapy with low-dose DNMTi to circumvent chemoresistance development during AML treatment, namely through marked shaping of the underlying clonal dynamics and transcriptomic landscape.

\section{Results}

Chemotherapy + DAC combination prevents hAML chemoresistance. In order to model the development of chemoresistance in hAML cells and assess its underlying clonal dynamics, we established an in vitro system using barcoded hAML cell lines (HEL and OCI-AML3) and exposed them to standard chemotherapeutic regimens optimized in order to significantly deplete viable cells (over 99\% elimination). Additionally, to model disease recurrences observed in chemotherapy-treated AML patients, we tested chemotherapeutic regimen doses that allowed barcoded hAML cell regrowth post-therapy exposure, thus generating in vitro relapses (Fig. 1a). A key aspect of this system is that lineage-tracing using DNA barcodes (BCs) allows the quantitative tracking of cell populations derived from each initially barcoded single-cell ${ }^{29}$, defined as barcoded clones (BC-clones), thus allowing quantification of BC-clonal dynamics in response to chemotherapeutic regimens. Barcoded hAML cells lines were exposed for $72 \mathrm{~h}$ to regimens of standard chemotherapy (doxorubicin-Doxo: $1.8 \mu \mathrm{M}$ and cytarabine-Cyta: $6 \mu \mathrm{M}$ ) alone or simultaneously combined with a low-dose of hypomethylating agent decitabine (DAC $0.1 \mu \mathrm{M}$ ). Viable cell numbers were monitored from treatment initiation (T0) until the time point when post-therapy cell cultures reached equivalent numbers as T0, termed Trelapse. We observed that Doxo, Doxo + Cyta and Doxo + Cyta + DAC regimens strongly depleted barcoded hAML cells in both cell lines (for example, depletion of $99.56 \%( \pm 0.23 \%$ eliminated cells, $n=6), 99.96 \%$ $( \pm 0.020 \%, n=8)$ and $99.90 \%( \pm 0.070 \%, n=8)$ HEL cells, respectively), whereas DAC single treatment had minor effects on cell numbers (Fig. 1b). Despite the high levels of cell elimination, Doxo \pm Cyta and Doxo + Cyta + DAC treated cells were able to re-grow back to the initial cell number within 30-35 days after treatment initiation. Next, to assess cell-intrinsic gain of chemoresistance in Trelapse cell populations, we re-exposed Trelapse AML cells to chemotherapy (Doxo + Cyta) for $72 \mathrm{~h}$. Strikingly, whereas chemoexposed (Doxo \pm Cyta) AML cells showed gain of resistance, upfront co-exposure to low-dose DAC preserved sensitivity to chemotherapy re-treatment, similarly to the no-treament (NT) control group (Fig. 1c). In agreement, Doxo \pm Cyta Trelapse samples showed a significant (2-4-fold) increase in IC50 values for doxorubicin compared to either NT or Doxo + Cyta + DAC relapse samples (Table 1). To confirm the capacity of upfront DAC combination to prevent chemoresistance development, we re-treated Trelapse samples with a new round of chemotherapy and quantified the frequency of Trelapse samples (Doxo, Doxo + Cyta and Doxo + Cyta + DAC) that generated a second relapse. We observed that 
a

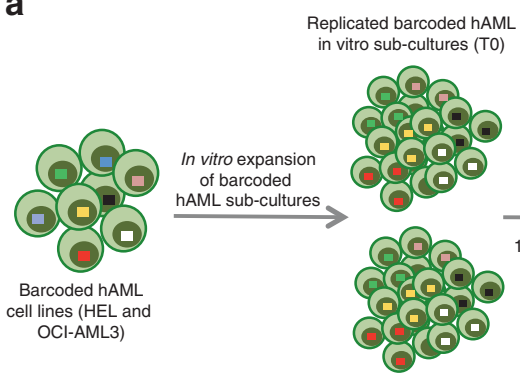

C

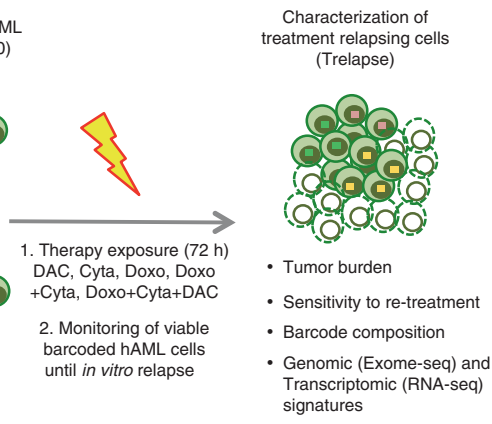

treatment relapsing cells

- Tumor burden

- Barcode composition

Genomic (Exome-seq) and signatures

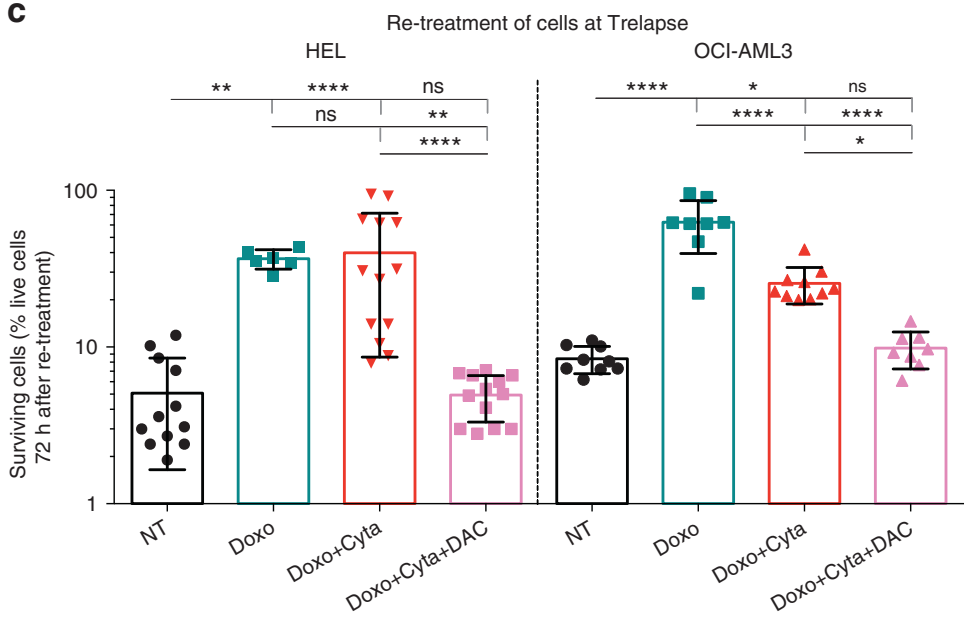

b

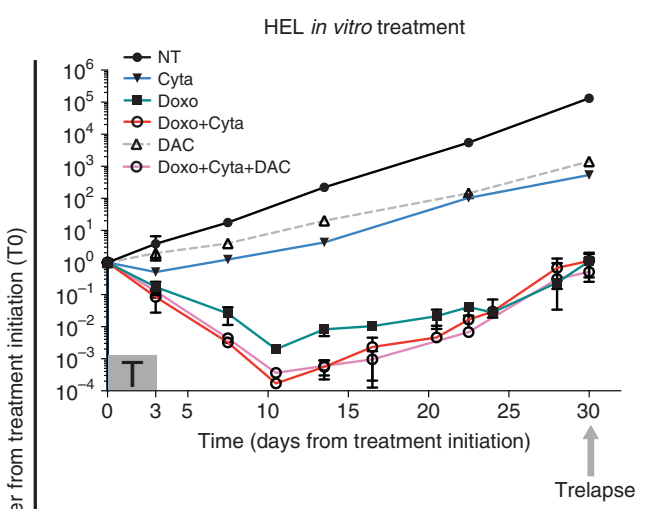

OCI-AML3 in vitro treatment

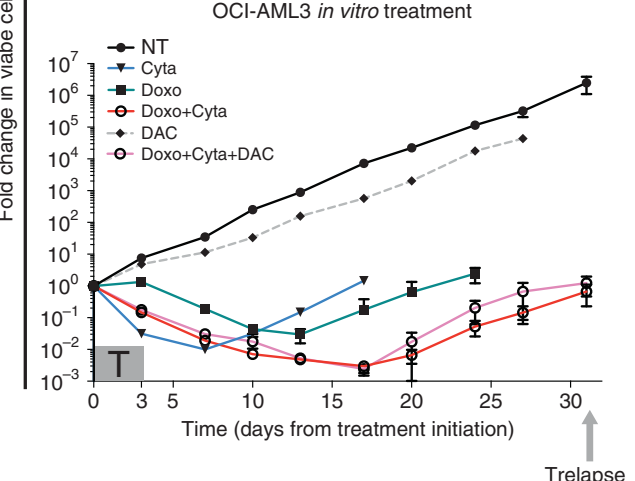

Fig. 1 Chemotherapy + Decitabine combination prevents chemoresistance in hAML cells. a Schematic diagram of the experimental system used. TO indicates the time of treatment initiation and Trelapse is defined as the post-therapy time point when re-grown hAML reached equivalent numbers as TO. NT-no treatment; DAC-decitabine $(0.1 \mu \mathrm{M})$; Doxo-doxorubicin $(1.8 \mu \mathrm{M})$; Cyta-cytarabine $(6 \mu \mathrm{M})$. b Total number of viable HEL and OCl-AML3 barcoded cells between TO and Trelapse, defined as a fold variation of cell number at each measured time point relatively to TO $(n=3$ for NT and DAC, $n=5$ for Cyta and Doxo, $n=8$ for Doxo + Cyta and Doxo + Cyta + DAC, independent replicates). T box indicates the period of therapy exposure. c Frequency of viable Trelapse HEL and OCI-AML3 barcoded cells (NT: $n=12 / 9$, Doxo: $n=6 / 8$, Doxo + Cyta: 13/10 and Doxo + Cyta + DAC: 13/8, independent replicates in HEL / OCl-AML3 respectively) after re-exposure to Doxo + Cyta for $72 \mathrm{~h}$. Concerning panels $b$ and $\mathrm{c}$ displayed graphs show mean \pm s.d.; $P$ values were determined by one-way ANOVA test. ns-not significant, ${ }^{\star} P<0.05 ;{ }^{\star \star} P<0.01 ;{ }^{\star \star \star} P<0.001 ;{ }^{\star \star \star \star} P<0.0001$. Source data are provided as a Source Data file

Table 1 Doxorubicin IC50 values of Trelapse hAML cells

\begin{tabular}{|c|c|c|c|c|c|}
\hline & \multirow[b]{2}{*}{ Cell line } & \multicolumn{4}{|c|}{ Trelapse Samples } \\
\hline & & NT & Doxo & Doxo + Cyta & Doxo + Cyta + DAC \\
\hline $\mathrm{IC} 50(\mu \mathrm{M})(n=3)$ & $\begin{array}{l}\text { HEL } \\
\text { OCl-AML3 }\end{array}$ & $\begin{array}{l}0.345 \pm 0.0352 \\
1.31 \pm 0.00503\end{array}$ & $\begin{array}{l}1.40 \pm 0.422 \\
4.20 \pm 0.256\end{array}$ & $\begin{array}{l}1.44 \pm 0.185 \\
3.80 \pm 0.351\end{array}$ & $\begin{array}{l}0.571 \pm 0.0242 \\
1.40 \pm 0.335\end{array}$ \\
\hline$P$-value ( $t$-test, relative to NT) & $\begin{array}{l}\text { HEL } \\
\text { OCl-AML3 }\end{array}$ & $\begin{array}{l}\text { NA } \\
\text { NA }\end{array}$ & $\begin{array}{l}0.0125 \\
<0.0001\end{array}$ & $\begin{array}{l}0.0005 \\
0.0003\end{array}$ & $\begin{array}{l}0.0008 \\
0.672\end{array}$ \\
\hline IC50 ratio (relative to NT) & $\begin{array}{l}\text { HEL } \\
\text { OCl-AML3 }\end{array}$ & $\begin{array}{l}1 \\
1\end{array}$ & $\begin{array}{l}4.06 \\
3.20\end{array}$ & $\begin{array}{l}4.20 \\
2.90\end{array}$ & $\begin{array}{l}1.66 \\
1.07\end{array}$ \\
\hline
\end{tabular}

while $100 \%$ of Doxo \pm Cyta Trelapse samples relapsed a second time, only 25\% (1 out of 4 ) of Doxo + Cyta + DAC Trelapse samples was able to relapse to re-treatment (Supplementary Fig. 1a, b). Importantly, by comparing the number of live cells at an equivalent time point (11 days) after treatment and retreatment, we observed that Doxo \pm Cyta Trelapse samples were significantly less sensitive to re-treatment, further confirming increased chemoresistance in these groups (Supplementary Fig. 1c). Overall, these results show that in vitro upfront combination of chemotherapy with low-dose DAC prevents the emergence of chemoresistant hAML cells.
Chemotherapy selects for a pre-determined set of BC-clones. To explore the clonal dynamics resulting from different treatment regimens we evaluated the $\mathrm{BC}$-clonal composition of $\mathrm{T} 0$ and Trelapse samples (Supplementary Fig. 2a-d). In the absence of therapy (NT), we observed stable and highly correlated (pearson correlation coefficient $>0.7$ ) BC-clone frequencies at day 30 relatively to $\mathrm{T} 0$ and also between replicates at Trelapse, even after $>10^{5}$-fold expansion (Supplementary Fig. $2 \mathrm{c}-\mathrm{j}$ ). This validates the clonal stability of our system in the absence of therapeutic pressure, thus allowing us to attribute $\mathrm{BC}$ clonal variations to therapeutic selection (rather than stochasticity of the system). Among 
a

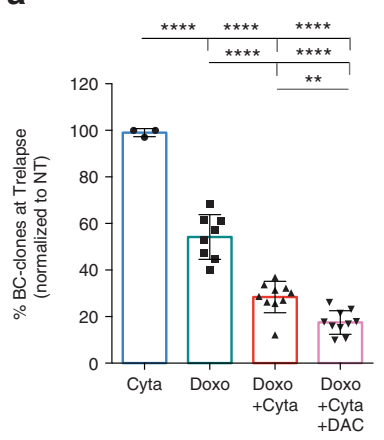

b

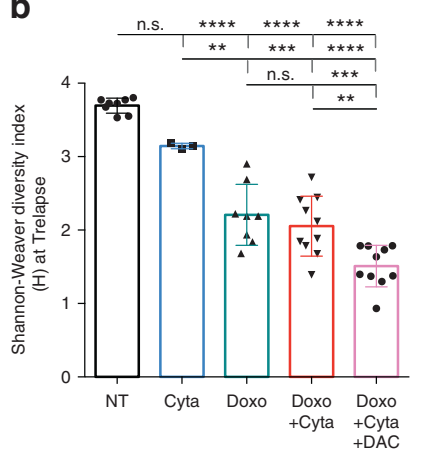

C

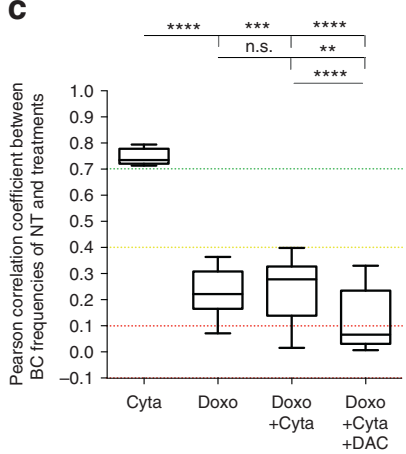

d

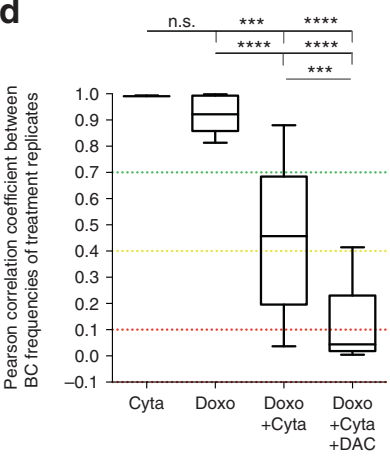

e

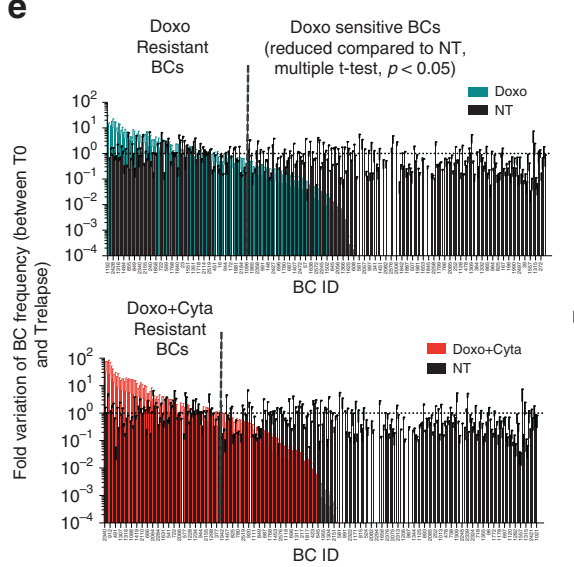

g

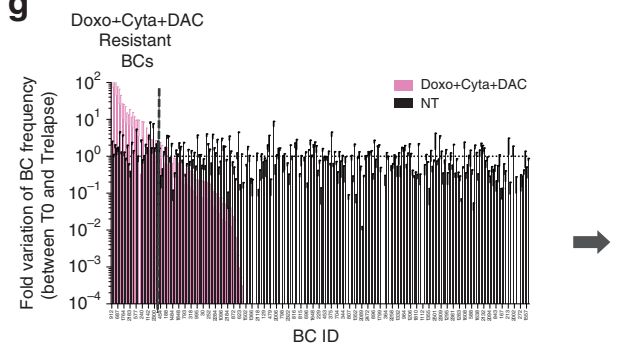

f

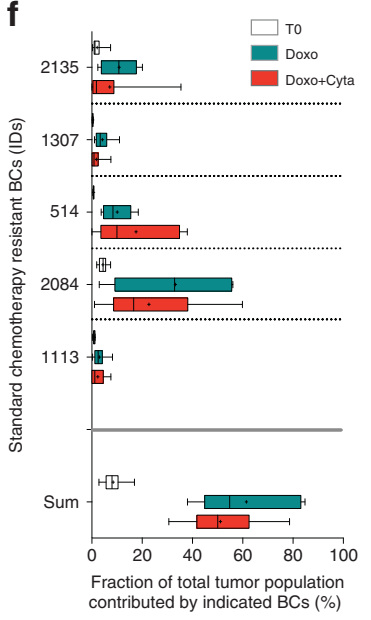

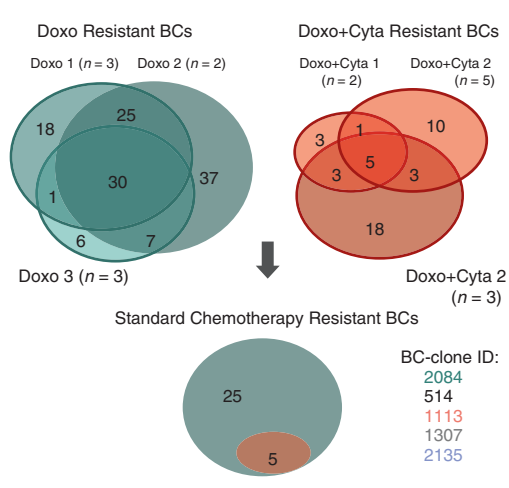

Doxo+Cyta+DAC Resistant BCs

Doxo+Cyta+DAC $1(n=2) \quad$ Doxo+Cyta+DAC $2(n=5)$

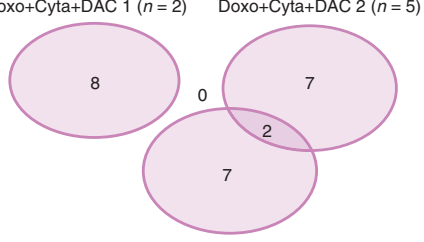

Doxo+Cyta+DAC $3(n=3)$

Fig. 2 Pre-determined set of BC-clones associates with chemoresistant relapses. (Data relative to HEL cell line) a Normalized (to NT) frequency of barcodes detected at Trelapse in Cyta $(n=3)$, Doxo $(n=8)$, Doxo + Cyta $(n=10)$ and Doxo + Cyta + DAC $(n=10)$ groups. b Shannon-Weaver diversity index $(H)$ of indicated Trelapse groups. $H=-\operatorname{Sum}(x i L N x i)$, where $x i$ is the frequency of each BC-clone in the population. $H$ reflects the BC number and how evenly distributed these are in the population (higher $\mathrm{H}$ results from higher $\mathrm{BC}$ number and more even distribution). c Pearson correlation coefficient between BC-clonal architectures of each treatment and NT groups (Cyta: $n=9$, Doxo: $n=19$, Doxo + Cyta \pm DAC $=36$ ). $\mathbf{d}$ Pearson correlation coefficient between BC-clonal architectures of replicates within each treatment group at Trelapse.(Cyta: $n=3$, Doxo: $n=10$, Doxo + Cyta \pm DAC $=14$ ) e Strategy used to define Doxo and Doxo + Cyta resistant BC-clones. On the left are depicted the fold variations of each BC-clone between TO and Trelapse in Doxo and Doxo + Cyta groups compared to NT, chemoresistant BC-clones were defined has the ones showing equal or increased fold variation relative to NT, determined by preforming multiple $t$-testing (per individual $B C, P<0.05)$. This was performed in three independent experiments $(n=2, n=5, n=3$ ). On the right, venn diagrams depicting the overlap of chemoresistant $B C$-clones, shared between all the replicates of three independent experiments for Doxo and Doxo + Cyta groups; as well as the overlap between these respective cores. The list of the chemoresistant BC-clones is indicated with respective BCidentification (id). f Average frequency of each indicated BC-clone (and their summed frequency) in Doxo $(n=8)$, Doxo + Cyta $(n=10)$ at Trelapse and TO $(n=5)$. Boxplots $\mathbf{c}, \mathbf{d}$ and $\mathbf{f}$ : center line is median, lower box bound is the $25 \%$ percentile, upper box bound is the $75 \%$ percentile, whiskers are minimum and maximum values. Cross is the mean $(\mathbf{f})$. $\mathbf{g}$ Same as $\mathbf{e}$ but applied to Doxo + Cyta + DAC group. Graphs of mean \pm s.d., $P$ values were determined by one-way ANOVA test. ns-not significant, ${ }^{\star} P<0.05$; ${ }^{\star \star} P<0.01$; ${ }^{\star \star \star} P<0.001 ;{ }^{\star \star \star \star} P<0.0001$. Source data are provided as a Source Data file

the Trelapse samples that were significantly impacted by therapy (Doxo, Doxo + Cyta, Doxo + Cyta + DAC), chemosensitive hAML cells relapsing to Doxo + Cyta + DAC combination showed lowest BC numbers and diversity (lowest ShannonWeaver diversity index H) (Fig. 2a, b, Supplementary Fig. 3a-c) which reflected in clonal architectures most divergent from NT samples (Fig. 2c, Supplementary Fig. 3d). By evaluating correlations between the $\mathrm{BC}$ architectures across replicates of each treatment at Trelapse, we found that $\mathrm{BC}$ distributions across Doxo relapses were highly reproducible (pearson $>0.7$ ) while addition of Cyta decreased the similarity of replicates, and further combination with DAC effectively abrogated all correlations (pearson <0.1) (Fig. 2d, Supplementary Fig. 3e). This suggests that DAC combination poses a stronger selective pressure on the system towards reducing the BC-clonal diversity and leading to relapses mediated by unpredictable (non-shared) BC-clones. To 
test if this effect was not the direct result of higher cell elimination (significantly different between Doxo and Doxo + Cytav \pm DAC groups), we preformed drug dose titrations that led to different cell elimination levels and assessed the corresponding BC-clone numbers at Trelapse. We observed a positive correlation between the number of live cells (at maximum selection point) and the equivalent number of detected BC-clones in each treatment titration, with the highest correlation coefficient observed in the Doxo + Cyta + DAC group (Supplementary Fig. 4a). Additionally, we compared Doxo + Cyta \pm DAC groups with a group receiving 9-fold higher doxorubicin concentration (Doxo9x). We confirmed that under equal cell elimination levels, the Doxo + Cyta + DAC group showed higher BC-clone elimination and, contrarily to the other groups, remained chemosensitive (Supplementary Fig. 4b-d). These data suggest that the level of cell elimination drives $\mathrm{BC}$-clone elimination in all conditions, but DAC combination selectively shows an increased capacity to deplete BC-clones even upon normalization of cell elimination levels. Next, the higher correlation between replicates in Doxo \pm Cyta treated samples compared to the Doxo + Cyta + DAC group prompted us to investigate if chemoresistant relapses shared a common set of BC-clones. For this, we analyzed the fold variation of each individual barcode frequency between T0 and Trelapse and based on statistical significance (multiple $t$-test) identified BC-clones with equal or increased frequency in Doxo \pm Cyta relapses compared to NT, which were defined as Doxo and Doxo + Cyta resistant BC-clones. By overlapping Doxo \pm Cyta resistant $\mathrm{BC}$-clones from three independent experiments, we identified a group of 5 or 6 BC-clones (in HEL and OCI-AML3, respectively) that were consistently selected in chemoresistant relapses (Fig. 2e, Supplementary Fig. 3g); and collectively constituted a major fraction of those Trelapse populations: $58,8 \%$ $( \pm 19.5 \%$ tumor fraction in Doxo/HEL, $n=8)$ and $50 \%( \pm 10.8 \%$ in Doxo + Cyta/HEL,$\quad n=10)$, which represented a $7-9$-fold expansion compared to T0 (Fig. 2f). Strikingly, the same analysis on Doxo + Cyta + DAC Trelapse samples revealed that no BCclone was consistently selected upon this treatment (Fig. 2g) further confirming the unpredictable nature of relapse upon DAC combination. Finally, to confirm the chemoresistance of Doxo \pm Cyta-selected BC-clone set, we evaluated its presence in second relapse (R2) samples after re-treatment. We observed that although R2 samples showed reduced BC-clone number compared to first relapse (R1) samples, their BC architecture was highly correlated with R1 (Supplementary Fig. 1d, e), suggesting a conserved BC composition to R1. Furthermore, there was an expansion of Doxo \pm Cyta resistant BC-clones summed frequency in R2 relatively to R1 (Supplementary Fig. 1f, g). Altogether, these data indicate that, whereas chemoresistant relapses associate with selection and expansion of a pre-determined set of BC-clones (that persist after two rounds of chemotherapy), DAC combination-driven chemosensitive relapses have reduced $\mathrm{BC}$ clonal diversity and are mediated by unpredictable BC-clones.

DAC combination selects unpredictable and unfit BC-clones. The ability of DAC combination to generate chemosensitive hAML relapses, led us to dissect the effects of DAC on hAML clonal dynamics. Concerning the effect of low-dose DAC as a single agent, we observed a mild reduction on genomic DNA methylated cytosine frequency, and a decrease in the population doubling time (Supplementary Fig. 5a, b), as previously described $^{30}$. At the BC level, these cells showed great similarity to NT samples and also between replicates (Supplementary Fig. 5c-e), suggesting that low-dose DAC alone has minimal impact on hAML BC-clonal dynamics. Next, we assessed whether DAC combination with chemotherapy impacted on the selection of the above-identified pre-determined set of chemoresistant BC-clones. By assessing BC-clonal compositions in the different experimental groups, we observed that, while the pre-determined BC set (with particular dominance of BC-clone 2084; dark green) was enriched in Doxo \pm Cyta relapses, it was strikingly underrepresented in Doxo + Cyta + DAC relapses (Fig. 3a). In agreement, these showed significantly reduced frequency of chemoresistant BC-clones in the top 3 most frequent BC-clones compared to Doxo \pm Cyta relapses (Fig. $3 \mathrm{~b}$ ). To improve our assessment of the effect of DAC combination on the pre-determined set of chemoresistant BC-clones, we quantified the frequency fold change of each of these clones between T0 and Trelapse under each treatment, and normalized it to the equivalent fold change observed in NT conditions-hereby defined as competitive index (CI). Strikingly, CI for the majority of the BC-clones was significantly reduced under the selective pressure of Doxo + Cyta + DAC compared to Doxo \pm Cyta (Fig. 3c, Supplementary Fig. 3h), suggesting that DAC combination impairs the chemotherapydriven selection of pre-determined chemoresistant BC-clones. To validate these findings with another hypomethylating agent, we evaluated the combination of chemotherapy with azacitidine (Aza). Like DAC, Aza also prevented chemoresistance while supressing pre-determined chemoresistant BC-clones (Supplementary Fig. 6a-g), strongly supporting the conclusion that this is a general effect of hypomethylating drugs. We next sought to characterize the alternative $\mathrm{BC}$-clones driving chemosensitive relapses upon DAC combination. Given the previously shown lack of a conserved set of BC-clones specifically selected by Doxo + Cyta + DAC, we focused our analysis of top 3 most frequent relapsing $\mathrm{BC}$-clones per culture. We observed that the most abundant BC-clones of Doxo + Cyta + DAC were rarer at T0 but underwent greater expansions compared to chemoresistant BCclones relapsing after chemotherapy alone (26 versus 7-13 fold, in HEL) (Fig. 3d, Supplementary Fig. 3i). Importantly, by assessing the fold change of the frequency of each individual BC-clone between T0 and Trelapse in NT conditions-hereby defined as fitness-we observed that the top 3 BC-clones of Doxo + Cyta + DAC had reduced fitness compared to those of Doxo \pm Cyta (Fig. 3e, Supplementary Fig. 3j), indicating that in the absence of therapy these rarer clones are outcompeted. As expected, the average $\mathrm{CI}$ of the top $3 \mathrm{BC}$-clones relapsing to Doxo + Cyta + DAC was significantly higher compared to those of Doxo \pm Cyta, confirming their gain in competitiveness specifically in the context of DAC combination (Fig. 3f). Finally, to assess if DAC addition impaired chemotherapy-driven BC-clone selection preferentially in the elimination or re-growth stages of our in vitro model, we preformed detailed longitudinal assessment of BC-clonal dynamics after treatment (Supplementary Fig. 7a). Focusing on the most dominant chemoresistant BCclone (2084), we observed that DAC combination had only a mild impact on the initial elimination stage, but strongly impacted on its capacity to re-grow (Supplementary Fig. 7b, c). By contrast, the less fit and rarer BC-clone 2252 was suppressed by Doxo + Cyta (Fig. 7d) but expanded massively and selectively under DAC combination (Supplementary Fig. 7e). Altogether, these data show that DAC combination suppresses the re-growth of chemoresistant clones, while favoring the selection and massive expansion of rarer and less fit clones that remain sensitive to chemotherapy re-treatment.

DAC shapes the transcriptome and function of hAML relapses. Having established the impact of DNA hypomethylating agents on hAML clonal dynamics underlying chemoresistance development, we next sought to decipher its molecular basis. In order to clarify the contribution of genetic and transcriptomic 
a
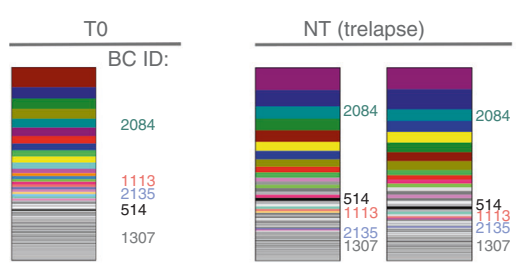

Doxo (trelapse)

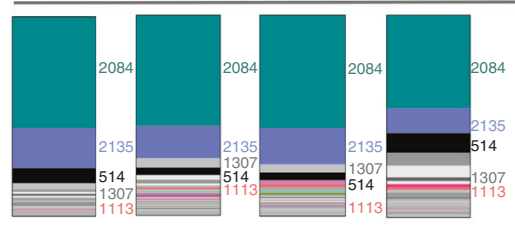

Doxo + Cyta (trelapse)

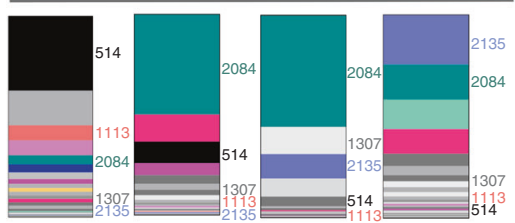

Doxo + Cyta + DAC (trelapse)

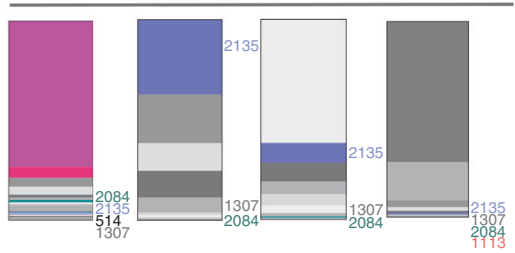

b

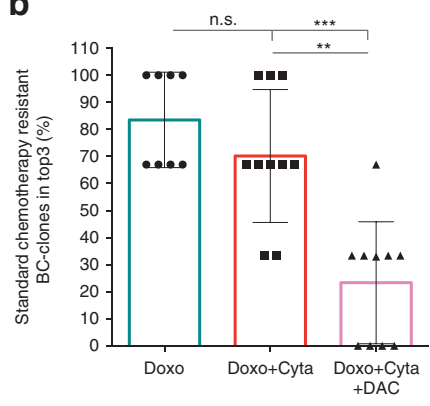

d

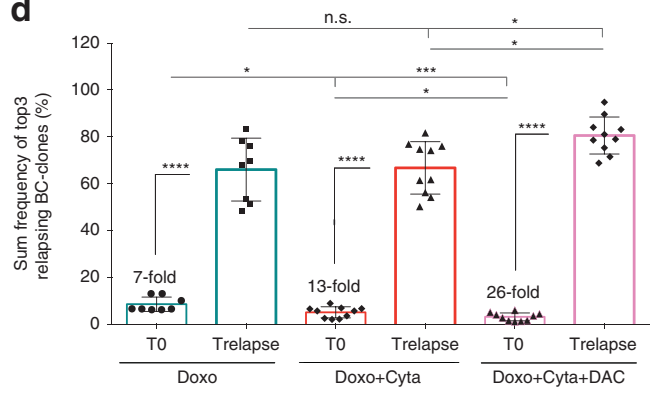

$\mathbf{f}$

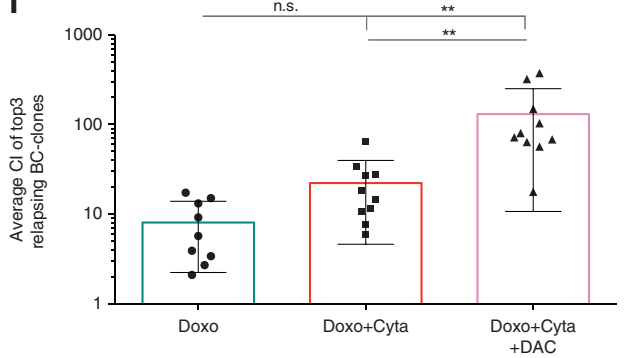

C

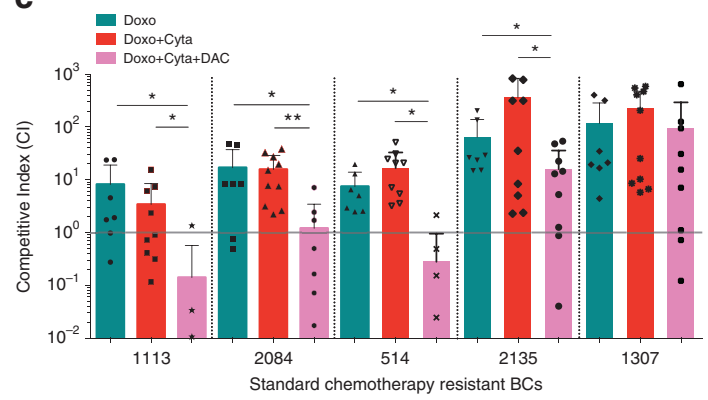

e
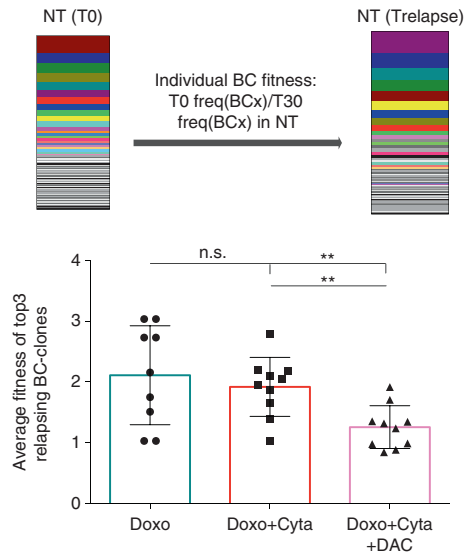

Fig. 3 Decitabine combination suppresses the re-growth of chemoresistant $B C$-clones. (Data relative to $H E L$ cell line) a $B C$-clonal composition of representative NT, Doxo, Doxo + Cyta and Doxo + Cyta + DAC samples at Trelapse. Each BC-clone has a fixed color-code across all samples. Chemoresistant BC-clones are indicated. $\mathbf{b}$ Average frequency at which chemotherapy-resistant BC-clones constitute the top 3 most dominant clones at Trelapse. In total, 3 out of 3 represents $100 \%$ frequency, 2 out of 3: 67\%, 1 out of 3: 33\% and 0 out of 3: $0 \%$ (Doxo: $n=8$, Do33xo + Cyta \pm DAC: $n=10$ ). c. Competitive index (Cl) of each chemoresistant BC-clone in Doxo $(n=8)$, Doxo + Cyta $(n=10)$ and Doxo + Cyta + DAC $(n=10)$ groups. A Cl of 1 (dotted line) represents a similar barcode fold variation form TO to Trelapse in the indicated condition relative to NT. d Summed frequency of top 3 most dominant BC-clones in each treatment group at Trelapse and their matched frequency at TO. The fold variation of the summed frequency of these BC-clones between TO and Trelapse is indicated. e. Schematic representation of experimental approach to calculate BC-clone specific fitness. Lower graph-Average fitness of the top 3 most dominant $\mathrm{BC}$-clones from indicated groups (Doxo: $n=9$, Doxo + Cyta \pm DAC: $n=10$ ). $\mathbf{f}$ Competitive index $(C l)$ of the top 3 most dominant BC-clones in indicated groups at Trelapse (Doxo: $n=9$, Doxo + Cyta \pm DAC: $n=10$ ). Graphs of mean \pm s.d., $P$ values were determined by t-test (panel c.)one-way ANOVA test. ns - not significant, ${ }^{\star} P<0.05 ;{ }^{\star \star} P<0.01 ;{ }^{\star \star \star} P<0.001 ;{ }^{\star \star \star \star} P<0.0001$. Source data are provided as a Source Data file

determinants in our model, we performed exome- and RNAsequencing on Trelapse samples. We observed an overall stability of the genomic composition of NT, Doxo \pm Cyta and Doxo + Cyta + DAC samples (Supplementary Fig. 8a-e), corroborated by high correlation between exonic-variant frequencies (in total exome and in AML/cancer gene panels) in the different groups (Supplementary Fig. 8f, g). Despite the overall sharing of exomic variants between all the conditions, we observed exonic variants unique to each condition (Supplementary Fig. 8c), suggesting an active on-going genetic clonal evolution process in our experimental model. However, few of these variants were on genes thought to have a causative role in AML pathogenesis ${ }^{13}$, the exception being de novo subclonal FLT3 mutations of unknown functional consequence and thus likely representing passenger mutations in Doxo + Cyta + DAC relapses (Supplementary Fig. 8f). On the contrary, established AML driver mutations $J A K 2^{V 617 F}$ and $P 53^{M 133 K}$ (P53 loss of function) were present at variant allele frequencies of $100 \%$ in all groups, as expected from their role as founding mutations in HEL cell line $e^{31}$
(Supplementary Fig. 8f). Importantly, there were no common mutations exclusively in chemoresistant groups (Doxo and Doxo + Cyta) in genes known to be mutated in chemoresistance ${ }^{11}$. These observations suggest that factors other than exomic mutation could participate in chemotherapy resistance. To further explore this hypothesis, we preformed transcriptomic analysis on Trelapse samples. Contrary to exomic data, but in agreement with BC-clonal dynamics, RNA sequencing revealed that Doxo + Cyta + DAC samples were clearly more divergent from NT than Doxo \pm Cyta groups (Fig. 4a), showing the highest number of statistically significant differentially expressed genes relative to NT (Doxo: 188; Doxo + Cyta: 40; Doxo + Cyta + DAC: 1049 -genes with fold change $><$ than \pm 3 ; adjusted $p$-value $<0.01)$. Doxo and Doxo + Cyta groups showed a conserved transcriptomic profile, supporting their previously established BC-clonal and functional convergences (Fig. 4a,b). Interestingly, gene set enrichment analysis (GSEA) revealed that Doxo + Cyta + DAC relapses were enriched for cell proliferation pathways (E2F and MYC target genes) and DNA synthesis (Fig. 4b,c). We 
a

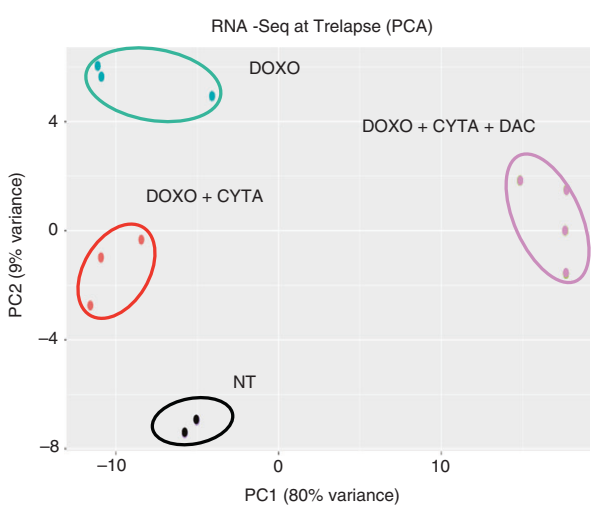

b

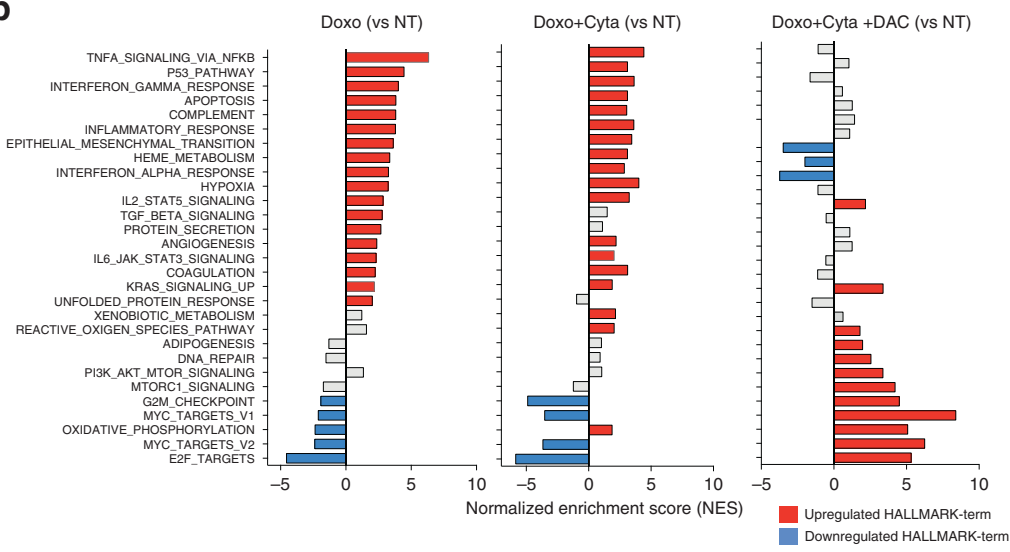

C

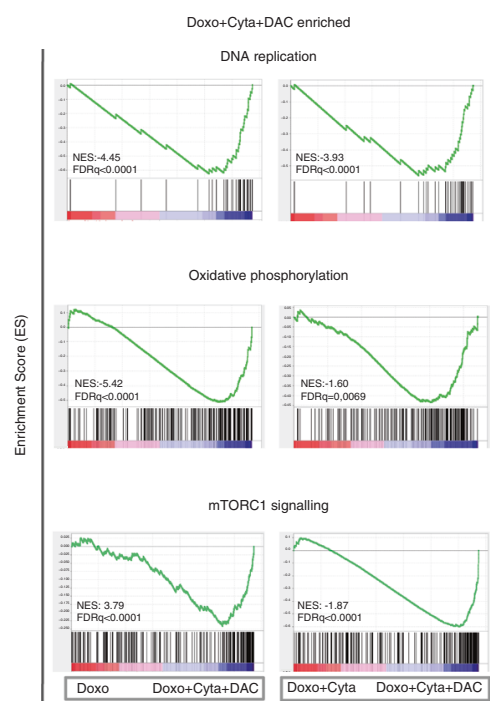

$\mathbf{h}$

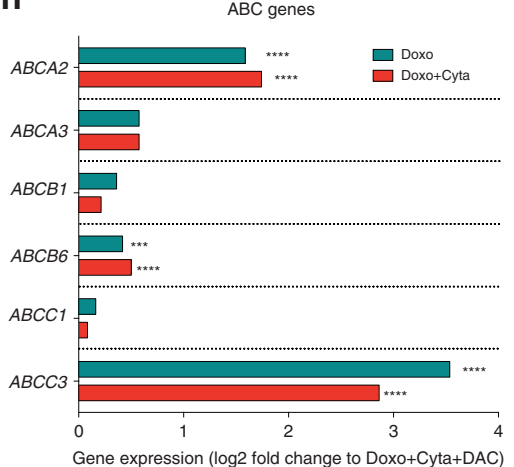

d

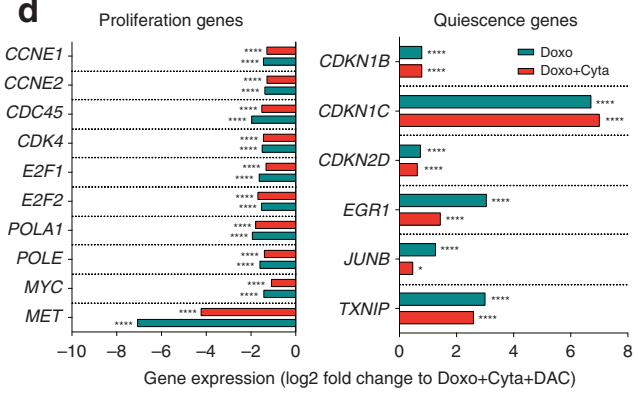

f

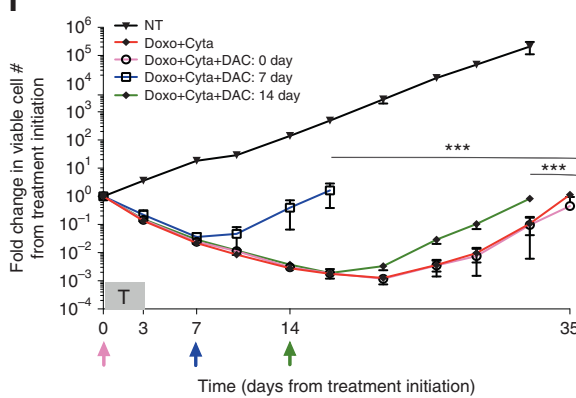

i

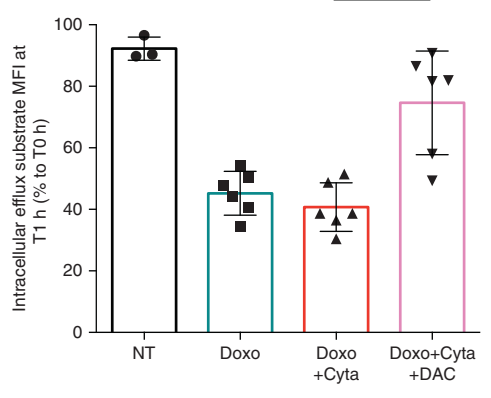

e

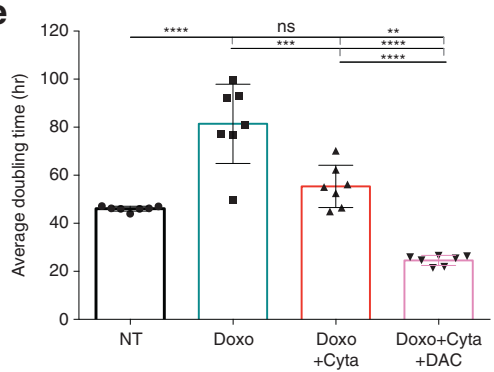

g
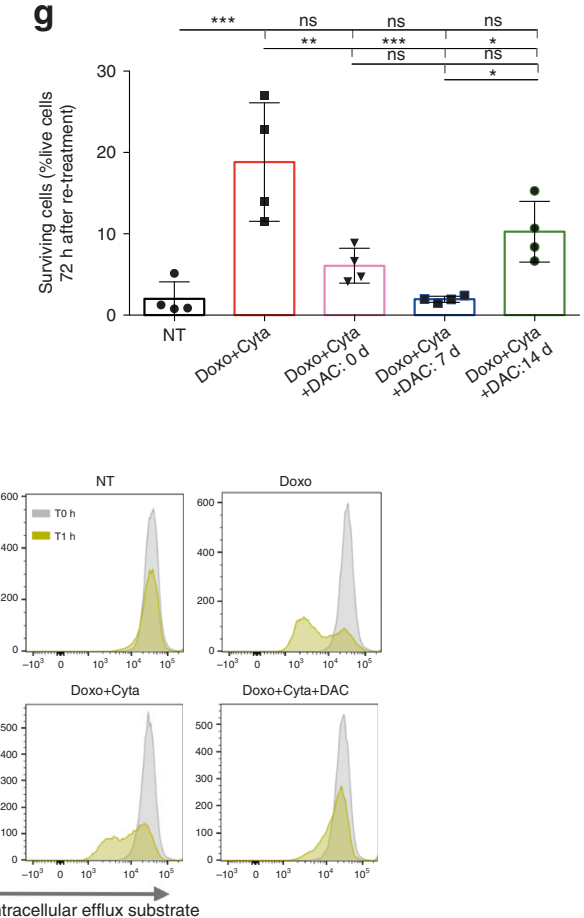

also observed increased expression of pathways related to metabolism such as mTORC1 signaling ${ }^{32}$ and oxidative phosphorylation, suggesting an overall increase in proliferative and biogenesis capacity of Doxo + Cyta + DAC relapses compared to both NT and Doxo \pm Cyta groups, further reflecting the higher clonal expansion observed in Doxo + Cyta + DAC relapses. In strike contrast, Doxo \pm Cyta samples showed not only a decrease in proliferative pathways, but an enrichment in pro-inflammatory pathways together with an up-regulation of the hypoxia pathway
(Fig. 4b,c), suggestive of a quiescence state in Doxo \pm Cyta relapses ${ }^{33,34}$. This was further supported by the finding that multiple genes involved in cellular quiescence such as CDKN1b, CDKN2d, EGR-1 or TXNIP were enriched in Doxo \pm Cyta relapses (Fig. $4 \mathrm{~d}$ ). Strikingly, the most enriched transcript relative to Doxo + Cyta + DAC was CDKN1C, a key inhibitor of several G1 cyclin/Cdk and negative regulator of cell proliferation that directly mediates stem-cell quiescence ${ }^{35,36}$ (Fig. 4d, Supplementary Figure 9a). Conversely, multiple genes involved in cellular 
Fig. 4 Dectabine shapes the transcriptome and proliferative capacity of relapsing hAML. (Data relative to HEL cell line) a Principal component analysis plot of differential gene expression at Trelapse between experimental groups. b Gene set enrichment analysis (GSEA) of HALLMARK gene set terms significantly (FDRq < 0.05) upregulated (red) and downregulated (blue) in each treatment group as compared to NT, at Trelapse. Gray bars represent no statistically significant change. c GSEA of differentially expressed pathways in Doxo + Cyta + DAC group compared to Doxo \pm Cyta groups at Trelapse. For GSEA analysis Kolmogorov-Smirnov statistical test was preformed. d Differential expression of indicated proliferation and quiescence genes between indicated groups at Trelapse. e Doubling time (hours) of indicated groups at Trelapse $(n=7)$. $\mathbf{f}$ Fold change in total number of viable AML cells between day 0 and 35 after chemotherapy treatment. Arrows indicate time point of DAC ( $0.1 \mu \mathrm{M}$ ) addition: 0 (pink), 7 (blue), and 14 (green) ( $n=3$ ). Cells were in all cases exposed to DAC for $72 \mathrm{~h}$. $\mathbf{g}$ Frequency of viable cells after in vitro (re-)exposure to Doxo + Cyta for $72 \mathrm{~h}$ in NT, Doxo + Cyta and Doxo + Cyta + DAC ( 0,7 or 14 days) relapsing cells $(n=4)$. $\mathbf{h}$ Differential expression of indicated ATP-binding cassette transporters (ABC) genes between indicated groups at Trelapse. $\mathbf{i}$ Intracellular efflux substrate MFI of NT, Doxo \pm Cyta and Doxo + Cyta + DAC cells at Trelapse. MFI was determined after $1 \mathrm{~h}$ (T1h) at $37^{\circ} \mathrm{C}$ and depicted as the ratio of the respective MFIs measured immediately after intracellular efflux substrate staining at TOh ( $n=6$ in all groups except NT: $n=3$ ). Representative histograms. Graphs of mean \pm s.d. $P$ values were determined by one-way ANOVA test. ns - not significant, ${ }^{\star} P<0.05$; ${ }^{\star \star} P<0.01$; ${ }^{\star \star \star} P<0.001 ;{ }^{\star \star \star \star} P<0.0001$. Gene expression graphs $(\mathbf{d}, \mathbf{h})$ represent mean log2(fold) change and indicated adjusted $P$ value calculated by Wald testing. Source data are provided as a Source Data file

proliferation pathways such as cyclins (CCNE1,E2), E2F transcription factors $(E 2 F 1,2)$ and DNA polymerase subunits $(P O L A 1, P O L E)$ where highly expressed in Doxo + Cyta + DAC relative to Doxo \pm Cyta relapses (Fig. $4 \mathrm{~d}$ ). Furthermore, the tyrosine-protein kinase $M E T$, a driver of AML proliferation ${ }^{37}$ was among the highest expressed transcripts in Doxo + Cyta + DAC relapses. The increased proliferative capacity of Doxo + Cyta + DAC (compared to Doxo \pm Cyta) relapses was further substantiated by lower doubling times (Fig. 4e, Supplementary Fig. 9b), increased G2-S-M cell cycle stage frequency (Supplementary Fig. 10a, b), and consistently smaller fractions of undivided cells as assessed by CSFE labeling (Supplementary Fig. 10c). To confirm the ability of DAC to antagonize the quiescent state induced by chemotherapy, we added DAC $(0.1 \mu \mathrm{M})$ at days 7 or 14 after chemotherapy exposure, and observed markedly earlier hAML cell re-growth compared to chemotherapy alone (Fig. 4f). Critically, whereas Doxo + Cyta relapses acquired chemoresistance, relapses resulting from DAC combination at all tested time points remained as chemosensitive as NT samples (Fig. 4g), suggesting an overt positive association between quiescence disruption and chemosensitivity in our model. Next, to further dissect the mechanisms of chemoresistance we focused the analysis on genes implicated in AML response to chemotherapy. Although there were no differences in the majority of genes associated with doxorubicin or cytarabine resistance ${ }^{38}$, we observed that the $A B C G 2$ gene was significantly up-regulated in all treatment conditions compared to NT (Supplementary Data 1), suggesting that this gene family associates with relapse in our system. Further analysis of the ATP-binding cassette transporters $(A B C)$ gene family revealed that multiple $A B C$ genes implicated in AML relapse and chemoresistance ${ }^{39}$ were upregulated in chemoresistant Doxo \pm Cyta groups compared to Doxo + Cyta + DAC, particularly ABCC3, A2 and B6 (Fig. 4h). Strikingly this associated with increased transporter activity in the Doxo \pm Cyta groups compared to NT and Doxo + Cyta + DAC groups (Fig. 4i), further implicating $\mathrm{ABC}$ gene family in chemoresistance development in our system. Overall, these data demonstrate that chemoresistant Doxo \pm Cyta relapses display increased quiescence and $\mathrm{ABC}$ transporter activity phenotypes that are completely reversed upon DAC combination, leading to highly proliferative chemosensitive relapses. Importantly, these findings also suggest that DAC acts mainly via modulation of the transcriptomic landscape of relapsing hAML cells, rather than impacting on their genetic (mutational) profiles.

DAC combination reduces stemness properties of hAML relapses. Low proliferation and quiescent states in leukemic blasts have been mechanistically linked to resistance to cytotoxic drugs $^{40,41}$. Importantly, in AML such low cycling states have been tracked down to sub-populations of leukemic cells that possess enhanced self-renewal and leukemia-initiating capacity, termed leukemia stem cells (LSCs) ${ }^{42-44}$. In fact, LSC frequency and associated transcriptional signatures carry clinical prognostic impact and have been extensively associated with chemoresistance and leukemia relapse ${ }^{17-19,45-47}$. We thus hypothesized that DAC addition to chemotherapeutic regimens could impact leukemia stemness properties. Consistent with our hypothesis, multiple stem-cell signatures: adult tissue stem-cell ${ }^{48}$, hematopoietic $^{49}$, mesenchymal ${ }^{50}$ and cancer ${ }^{51,52}$ stem cell signatures, were all enriched in Doxo \pm Cyta compared to Doxo + Cyta + DAC relapses (Fig. 5a). Moreover, the expression of multiple genes associated with hematopoietic and leukemic stem cell selfrenewal (HoxA4,B4,B5; FoxO1,O3,O4; CBX7; ANG; EMCN) was significantly upregulated in Doxo \pm Cyta compared to Doxo + Cyta + DAC relapses (Fig. 5b). Furthermore, Doxo \pm Cyta relapses, but not Doxo + Cyta + DAC relapses, displayed a series of stem cell hallmarks: increased expression of LSC markers (\% CD34 + CD38- and CD99 MFI; Fig. 5c, Supplementary Fig. 9c), increased ATP-binding cassette (ABC) transporter activity (Fig. 4i) and increased aldehyde dehydrogenase activity (Fig. 5d). A key functional readout of leukemia cell stemness is their ability to initiate leukemia upon transplantation into irradiated immunodeficient mice ${ }^{53}$. To assess the frequency of leukemia-initiating cells (L-ICs) in Trelapse samples, these were engrafted in limiting dilution into sublethaly irradiated NRGS mice (Fig. 5e). Doxo + Cyta + DAC chemosensitive cells showed reduced L-IC frequency when compared to chemoresistant Doxo \pm Cyta relapses, leading to less aggressive leukemia development as reflected in increased overall survival of the hosts (Fig. 5f,g). Altogether, our functional assessment of Trelapse cell populations shows that chemoresistance development associates with lower proliferation and increased stemness properties, which are both prevented by up-front combination with DAC. Next, to assess functional leukemia-initiating capacity of individual BC-clones, we performed in vivo establishment of T0 samples with known BC architectures and L-IC frequency (Fig. 5h). By assessing in vivo engraftment of each individual $\mathrm{BC}$ in each individual mouse tested, we were able to define a group of $36 \mathrm{BC}$-clones with significantly higher leukemia-initiating capacity than the population average (HiL-IC BC-clones) (Fig. 5h,i). Quantification of HiL-IC BC-clones present in Trelapse samples confirmed that the majority was efficiently suppressed by DAC combination (Fig. 5j; Supplementary Fig. 9d). Importantly, among the HiL-IC HEL $\mathrm{BC}$-clones, we found four out of five pre-determined chemoresistant BC-clones previously identified in Doxo \pm Cyta Trelapses (Fig. 5k; Supplementary Fig. 9e), further demonstrating that chemoresistance strongly associates with in vivo leukemia initiating capacity at both population and BC-clonal levels. Finally, to confirm the ability of DAC combination to target L-ICs, we generated $\mathrm{BC}$-clones from in vivo established single-L-ICs (L-IC 
a

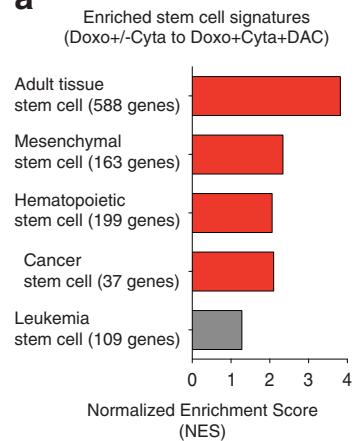

e

Leukemia growth for 10 weeks

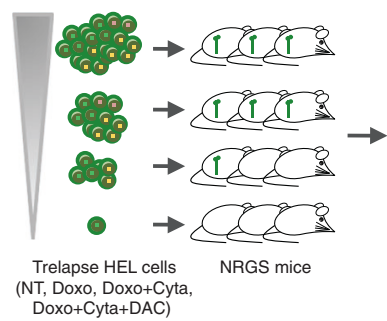

b

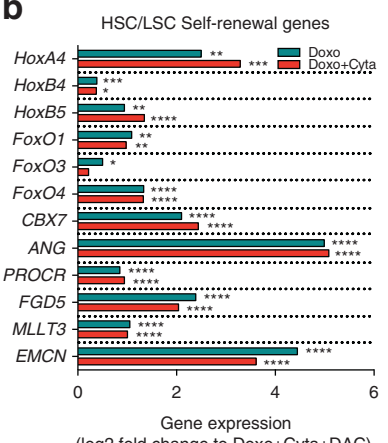

(log2 fold change to Doxo+Cyta+DAC)
C

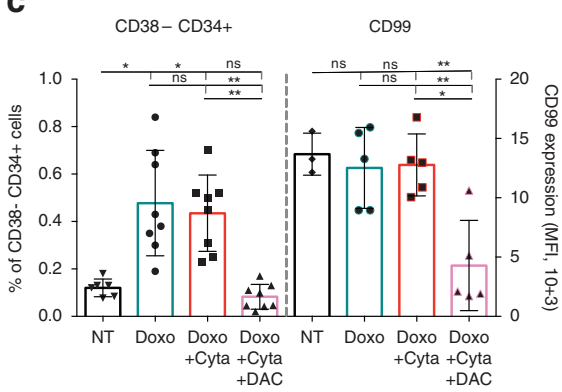

d

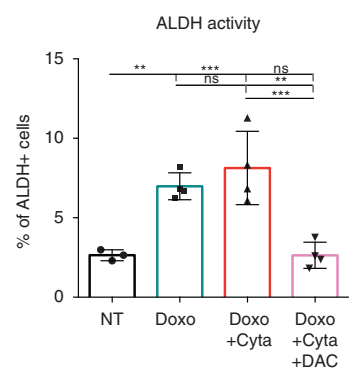

f

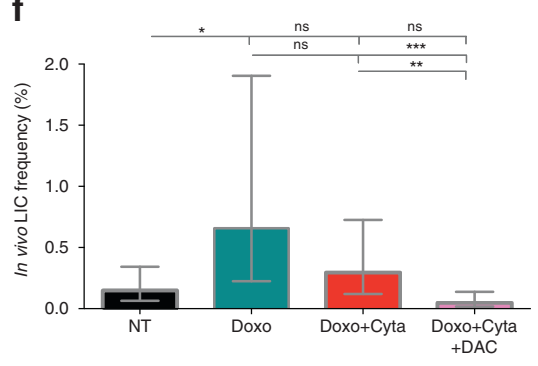

g

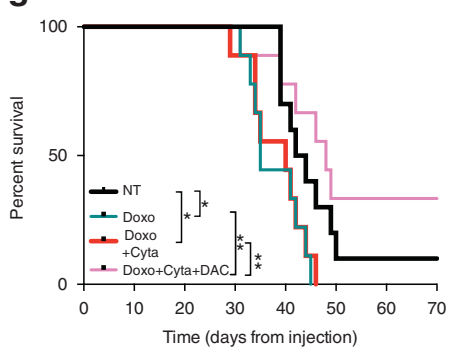

h

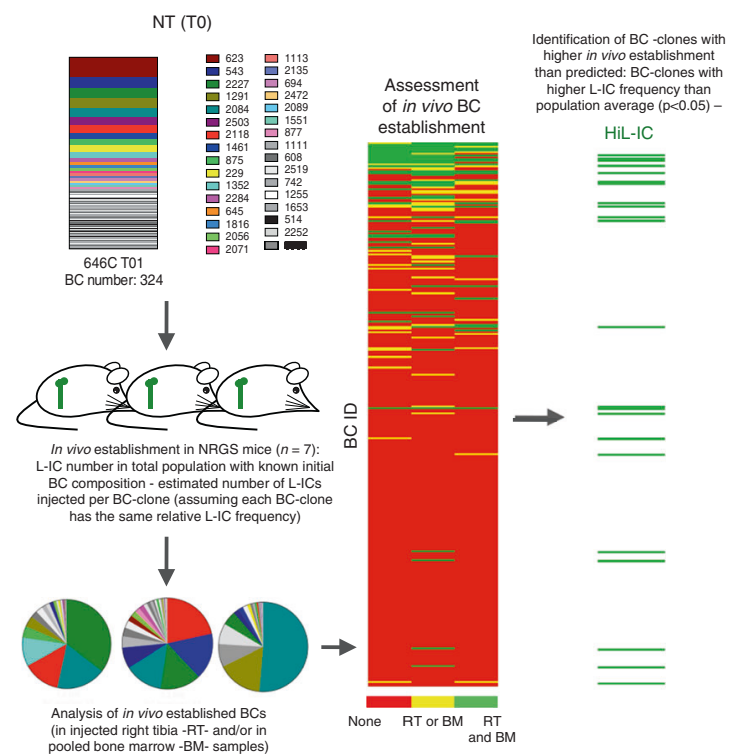

i HiL-IC BC-clones

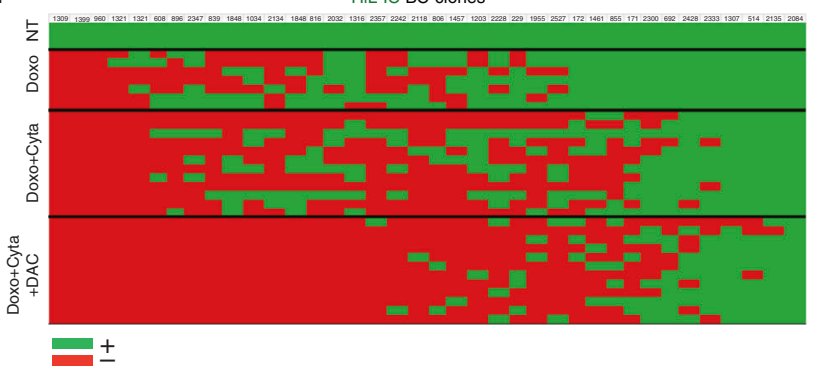

j

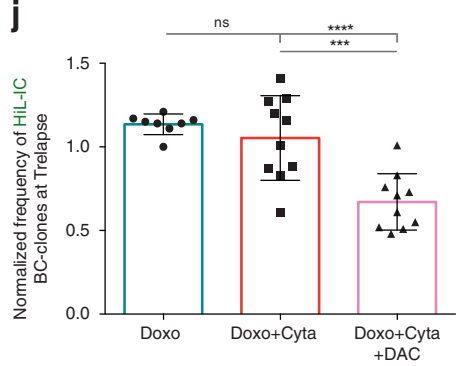

k

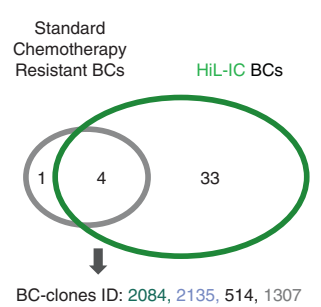

Fig. 5 Decitabine combination reduces stemness properties of relapsing hAML cells. (Data relative to HEL cell line) a GSEA of different stem-cell signatures significantly enriched (red, FDRq $<0.05$ calculated by Kolmogorov-Smirnov statistical test) in Doxo \pm Cyta Trelapses relative to Doxo + Cyta + DAC group (indicated NES are relative to the highest enrichment observed in Doxo \pm Cyta). b Differential expression of indicated genes implicated in hematopoietic and leukemic stem cells (HSCs, LSCs) self-renewal between indicated groups at Trelapse. c Frequency of CD38-CD34 + cells (left axis, NT: $n=6$, Doxo \pm Cyta and Doxo + Cyta + DAC: $n=8$ ) and CD99 mean florescence intensity (MFI) (right axis, NT: $n=3$, Doxo \pm Cyta and Doxo + Cyta + DAC: $n=5$ ) on indicated groups at Trelapse. d Fraction (\%) of cells with active ALDH enzymatic activity in indicated grups (NT: $n=3$, Doxo \pm Cyta and Doxo + Cyta + DAC: $n=4)$. e Schematic diagram of limiting-dilution assay performed with Trelapse populations from NT, Doxo \pm Cyta and Doxo + Cyta + DAC cells on NRGS mice, via intra-bone marrow injection of 10000, 1000, 100 and 10 cells. f Estimated leukemia-initiating cell (LIC) frequency in Trelapse populations from indicated groups (the y-axis denotes the confidence intervals-lower, estimate and upper for L-IC frequency). g Survival curves of mice transplanted with 1000 cells from Trelapse NT $(n=10)$, Doxo \pm Cyta $(n=9)$ and Doxo + Cyta + DAC $(n=9)$ samples. h Schematic diagram of the strategy used to identify BC-clones with a higher L-IC frequency than the population average (HiL-IC). $\mathbf{i}$ Heat-map scoring the presence (green) or absence (red) of HiLIC BC-clones (columns, total of 37) present in Trelapse populations from replicates of indicated groups (lines). j Normalized frequency of HiL-IC BC-clones present at Trelapse in indicated groups (Doxo: $n=8$, Doxo + Cyta \pm DAC: $n=10$ ). $\mathbf{k}$ Venn diagram depicting the overlap across BC-clones with HiL-IC and chemoresistant properties. Graphs of mean \pm s.d., $P$ values were determined by one-way ANOVA test. Survival curve analysis was performed by Log-rank (Mantle-Cox) testing. ns-not significant, ${ }^{\star} P<0.05 ;{ }^{\star \star} P<0.01 ;{ }^{\star \star \star} P<0.001 ;{ }^{\star \star \star \star} P<0.0001$. Gene expression graph (b) represents mean $\log 2$ (fold) change and indicated adjusted $P$ value calculated by Wald testing. Source data are provided as a Source Data file 
BC-clones)(Supplementary Figure 11a,b). In this setting, each BC-clone derives necessarily from an in vivo established L-IC. Strikingly, ex vivo treatment of in vivo established BC cells with Doxo + Cyta + DAC regimen significantly reduced the number of L-IC BC-clones compared to Doxo \pm Cyta (Supplementary Fig. 11c), thus confirming the ability of DAC combination to target L-ICs. Our data demonstrates that chemotherapy selects for pre-determined BC-clones with stemness properties, including low proliferation, chemoresistance and leukemia-initiating potential, which are suppressed upon DAC combination that thereby favors the expansion of chemosensitive BC-clones with decreased stemness capacity.

DAC combination depletes leukemia stem cells in hAML samples. Having established the capacity of upfront DAC combination to prevent stemness-mediated chemoresistance in our in vitro system, we aimed to validate this finding in a in vivo xenotransplantation model. For this purpose, we tested the effect of standard chemotherapy (Doxo + Cyta) alone or combined with DAC (Doxo + Cyta + DAC) on an in vivo orthotopic model of intra bone-marrow (BM) transplantation of hAML HEL cell line into NRGS mice (Fig. 6a) ${ }^{54}$. Contrarily to the in vitro system where more than $99 \%$ of the cells were eliminated by equivalent regimens (Fig. 1b), in vivo cell elimination in the peripheral blood of leukemic animals was mild (Fig. 6b). This resulted mainly from the inability to increase in vivo chemotherapy doses beyond the limit of chemotherapy toxicity in immunodeficient mice 55,56 . Notwithstanding this limitation, mice treated with Doxo + Cyta + DAC showed increased leukemia cell elimination and overall survival compared to untreated (NT), DAC alone and Doxo + Cyta treated mice (Fig. 6b,c). Next, to assess cell-intrinsic gain of chemoresistance, we sorted BM-resident hAML cells from non-treated (NT) and Doxo + Cyta \pm DAC treated mice and reexposed them to chemotherapy (Doxo + Cyta) ex vivo. Strikingly, whereas in vivo chemo-exposed AML cells showed a three-fold gain of resistance, co-exposure to DAC preserved NT-like sensitivity upon re-treatment (Fig. 6d). Despite the lower magnitude of chemoresistant gain in vivo compared to the in vitro system (likely resulting from the limited amount of chemotherapy used), these data confirmed the overall capacity of upfront DAC combination to prevent chemoresistance development in hAML cells. Importantly, by assessing the frequency of immunophenotypically defined LSCs (live CD38-CD34 + cells) in NT, Doxo + Cyta and Doxo + Cyta + DAC, we could confirm that DAC combination prevents the chemotherapy-induced increase in LSC frequency (Fig. 6e). Altogether, these data validate our in vitro observations on the effect of DAC combination on hAML stemness and chemoresistance at relapse in a more physiological in vivo xenotransplantation model. Finally, we tested the effect of DAC combination in targeting immunophenotypically defined LSCs (live CD33 + CD38- CD34 + cells) from human primary AML samples (Supplementary Figure 12; Supplementary table 1). Bone marrow AML blasts were cultured ex vivo in the presence of no treatment (NT), Doxo + Cyta or Doxo + Cyta + DAC for 3 consecutive days; and 3 days after drug withdrawal the absolute number of viable CD33 + CD38- CD34 + LSCs was assessed (Fig. 6f). We observed that while Doxo + Cyta depleted LSCs numbers in the majority of the samples, the combination with DAC clearly enhanced this effect, leading to a significant decrease in LSC numbers in 6 out 8 tested samples (samples 1, 3, 12, 15, 20, and 25; Fig. 6g,h). These data further strengthen our observations that upfront combination of low-dose decitabine with chemotherapy depletes LCS numbers in hAML relapses, thus attesting the potential of this approach to tackle stemnessassociated chemoresistance development in AML.

\section{Discussion}

Therapeutic resistance drives recurrences and represents a major hurdle to successful clinical management of cancer in general, and AML in particular. In this study we have established an experimental model system to characterize longitudinally the clonal dynamics and associated genetic and non-genetic determinants underlying chemoresistance development in hAML cells exposed to different chemotherapeutic regimens. Using in vitro lineage tracing coupled with exome, transcriptome and in vivo functional readouts we revealed the ability of low-dose DNMTis in upfront combination with chemotherapy to prevent chemoresistance development by suppressing the expansion of a predetermined set of AML clones with high stemness properties. These data represent a major advance to our understanding of the clinical benefits described for such combinatorial regimens in refractory/relapsed $\mathrm{AML}^{25-27}$.

The use of DNMTi to sensitize chemoresistant tumors has been applied in different cancer types with some success, having been associated with transcriptional activation of tumor suppressor genes such as TP53 $3^{57}$. Here, we found that combining chemotherapy with DAC leads to major transcriptomic and functional changes of relapsing hAML cells, leading to an overall decrease in stemness properties (quiescence, drug efflux capacity and leukemia-initiating/self-renewal capacity), which have been linked with poor clinical outcomes and chemoresistant relapse development in $\mathrm{AML}^{58}$. Among these properties, low proliferation and quiescent states in leukemic blasts have been directly associated with resistance to cytotoxic drugs, as chemotherapeutic agents preferentially target dividing cells ${ }^{40,41}$. Supporting this, we observed that concomitantly with multiple regulators of cellular quiescence, the CDKN1C gene, a key negative regulator of cell proliferation that directly mediates stem-cell quiescence and selfrenewal capacity in hematopoietic stem cells (HSCs) ${ }^{35,36}$, was the most enriched transcript in chemotherapy relapses when compared to DAC combination samples. High CDKN1C expression levels in BM associate with lower proliferative activity and poor survival after standard chemotherapy in both AML and MDS patients ${ }^{59}$. This further suggests that reduced proliferation in chemotherapy-driven relapses may represent a mechanistic pathway of resistance that is prevented by DAC addition, thus resulting in proliferative and chemosensitive relapses. Importantly DAC combination also associated with an overall decrease in the expression of multiple $\mathrm{ABC}$ gene family members (in particular $A B C C 3)$ which are well established regulators of chemoresistance in $\mathrm{AML}^{39,60}$. Another key transcriptional and functional aspect of chemoresistant hAML relapses in our model was increased leukemia-initiating capacity (in immunocompromised mice) and self-renewal gene expression, which was strikingly abolished upon DAC combination. This observation is in line with previous studies showing that DNA methylation promotes self-renewal and inhibits differentiation of both HSCs and LSCs; ${ }^{61,62}$ and that lowdose DNMT inhibitors can reduce the tumorigenicity of cancer stem cells in various models ${ }^{63}$. The molecular mechanism by which DNMTi combination impacted on the expression of stemness-associated genes was not established in our study. A potential explanation for the observed increase in proliferation upon DNMTi combination is the hypomethylation-dependent enhanced transcription of pro-cycling genes (e.g. $M E T^{64}$ ), whereas repressed signature genes (e.g. $C D K N 1 C, A B C C 3, C B X 7)$ may be indirectly regulated via hypomethylation-promoted transcription of their negative regulators. Critically, our study reveals for the first time the capacity of DAC in upfront combination with chemotherapy to reduce the stemness properties of hAML relapses, further encouraging the clinical assessment of upfront combination of low-dose DNMT inhibitors with standard chemotherapy as first line treatment of AML. 


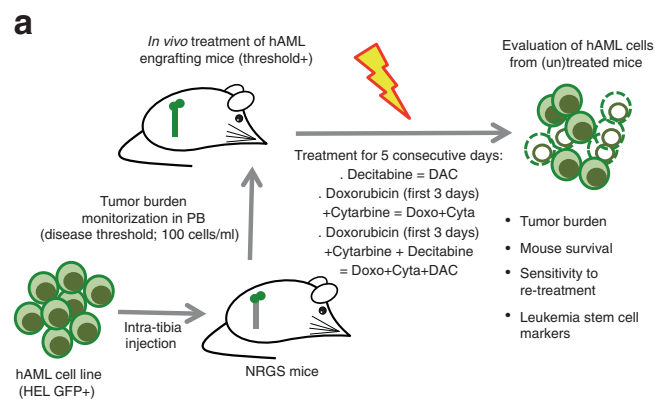

b

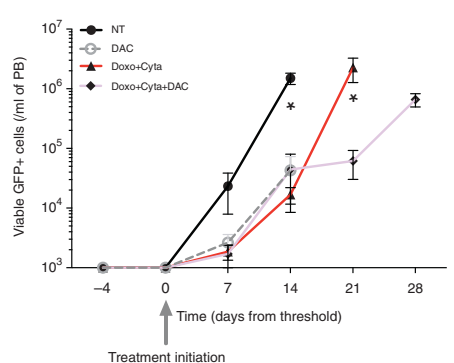

C

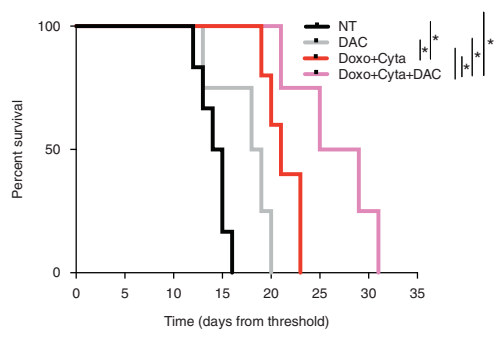

d

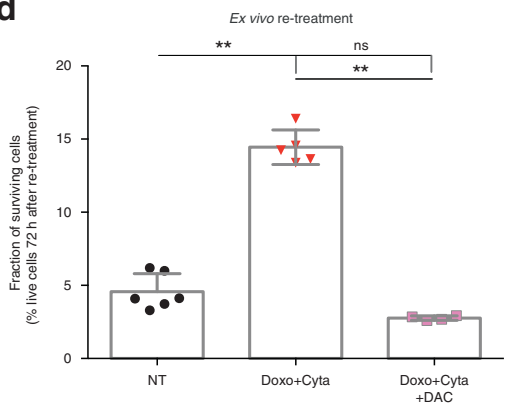

e

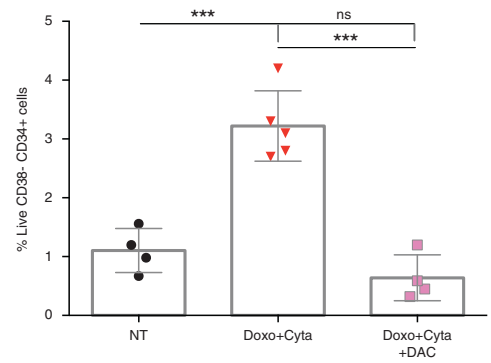

f

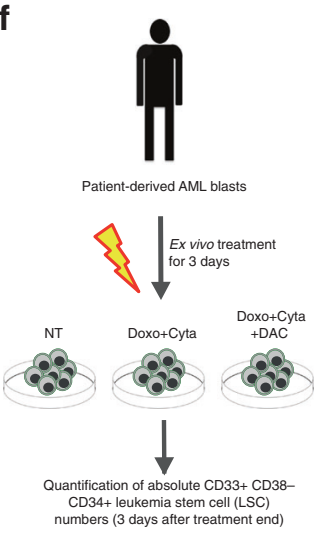

g

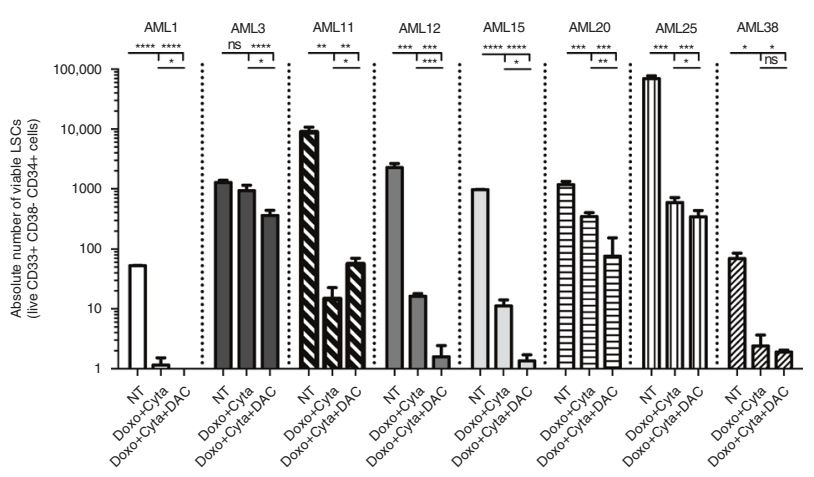

h

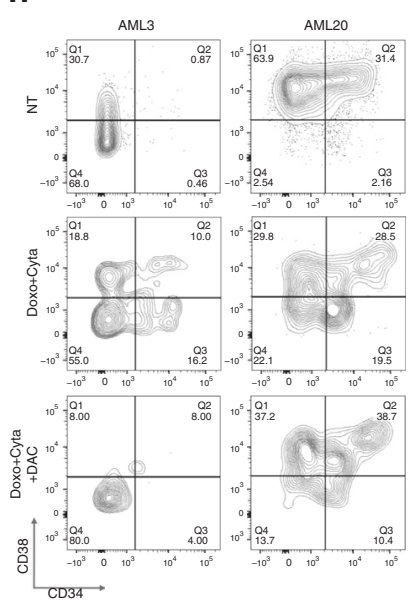

Fig. 6 Decitabine combination depletes LSCs from xenografts and AML patient samples. a Schematic diagram of the therapeutic setting used to treat NRGS mice bearing human AML (GFP + HEL) cells. Chemotherapy regimen (Doxo + Cyta): intraperitoneal (i.p.) injection of cytarabine (100 mg/kg per day over 5 days) and doxorubicin ( $3 \mathrm{mg} / \mathrm{kg}$ per day over the first 3days). Decitabine combination was initiated 2 days after Doxo + Cyta, via i.p. injection of 0,5 mg/kg DAC for 5 consecutive days. b The total number of viable hAML HEL (GFP +, 7AAD-) cells in peripheral blood (PB) and mouse survival (c) in untreated (NT, $n=6)$, DAC alone $(n=4)$, Doxo + Cyta $(n=5)$ and Doxo + Cyta + DAC $(n=4)$ groups. $\mathbf{d}$ Frequency of viable BM-sorted hAML cells, sorted from the BM of mice from the three groups described in $\mathbf{b}$, $\mathbf{c}$, after ex vivo exposure to Doxo + Cyta for $72 \mathrm{~h}$. e Frequency of CD38-CD34 + cells (left axis, $n=4-8$ ) on BM-sorted hAML cells from indicated groups. f Schematic diagram depicting ex vivo treatment of AML patient samples Doxo + Cyta and Doxo + Cyta + DAC and the subsequent quantification of immunophenotypically defined LSCs. g Absolute number of LSCs (live CD33 + CD38- CD34 + cells) after ex vivo exposure to Doxo + Cyta and Doxo + Cyta + DAC ( $n=3$ per sample). $\mathbf{h}$ Representative flow cytometry contour plots of CD38/CD34 staining gated on CD33 + VioletZombie- population. Graphs of mean \pm s.d., $P$ values were determined by one-way ANOVA test. Survival curve analysis was performed by Log-rank (Mantle-Cox) testing. ns-not significant, ${ }^{\star} P<0.05 ;{ }^{\star \star} P<0.01 ;{ }^{\star \star \star} P<0.001 ;{ }^{\star \star \star \star} P<0.0001$. Source data are provided as a Source Data file

To resolve the underlying clonal dynamics associated with the selection of chemoresistance we employed an in vitro system using barcoded hAML cells. In spite of its inherent limitation in modeling the complex biology of AML cells (e.g. bone marrow niche interactions ${ }^{65}$ ) this experimental setup offers two key advantages compared to in vivo systems: (1) drug doses are not limited by the sensitivity of immunocompromised mice to cytotoxic agents ${ }^{56}$; (2) stable steady-state clonal dynamics, which is lost upon in vivo xenotransplantation of human cancer cells into immunocompromised mouse models ${ }^{66}$ (our unpublished data), allowing for reproducible clonal dynamics assessment upon experimental perturbations. The latter is particularly relevant since, by creating replicates with a constant barcode composition, it allows testing the effect of different therapies on the same complex tumor population that would not be possible in the clinical setting. Using this unique feature of our system, we found a pre-determined set of $\mathrm{BC}$-clones that is consistently present in chemotherapy relapsing populations, indicating that, even in the absence of selective pressure, there are clonally related cells (BCclone) that share the molecular determinants that will lead to their selection upon chemotherapy exposure. Interestingly, molecular characterization of the mutational spectrum by exome 
sequencing showed a lack of genetic drivers for chemoresistance and an overall conserved exomic landscape in chemosensitive and chemoresistant groups. In stark contrast, transcriptomic assessment revealed a very dynamic shift in gene expression with a strong enrichment in stemness-associated pathways in chemoresistance hAML cells. Importantly, we observed that this predetermined set of chemoresistant BC-clones also displayed increased capacity to establish in in vivo immunocompromised mice (a classic LSC feature) prior to chemotherapy, indicating that transcriptional/functional defined stemness and chemoresistant states pre-exist in hAML BC-clones and are actively selected by chemotherapy. This indicates that hAML chemoresistance development pre-exists therapy exposure and results from transcriptomic rather than genetic selection, in line with recent studies implicating epigenetic reprogramming of transcriptomic heterogeneity at single-cell level in the development of therapeutic resistance, independently of genetic alterations ${ }^{67}$. Strikingly, our data revealed that upfront DNMTi combination impaired the selection of the chemoresistant pre-determined set of $\mathrm{BC}$-clones, and dramatically favored the expansion of a rarer set of clones that would be normally (i.e., in absence of this specific pressure) outcompeted. This was accompanied by a strong transcriptomic signature of proliferation pathways, further substantiating the clonal expansion observed in these populations upon relapse. These data strongly indicate that DAC uniquely affects the re-growth capacity of each individual BC-clone in a differential manner, causing a significant impairment on the regrowth capacity of pre-determined $\mathrm{BC}$-clones associated with chemoresistance, while favoring the expansion of rarer BC-clones that remain chemosensitive. Although the mechanistic basis of this differential impact of DAC on different BC-clones was not completely clarified here, it is very likely to depend on clonalspecific epigenetic states ${ }^{22}$. In fact, our data suggests a model where, in response to chemotherapy, hAML regrowth is heavily dependent on, and potentially driven by, LSCs-as shown by a clear enrichment of stemness at relapse. Given the high sensitivity of CSCs to DNMTis ${ }^{61-63}$ it is therefore possible that in the presence of DNMTi relapses no longer derive from LSCs, but are now largely driven by cells with lower stemness that regenerate the leukemia via activation of proliferative pathways while remaining sensitive to chemotherapy.

In summary, our study demonstrates the ability of low-dose DNMTi in combination with chemotherapy to dramatically shape AML clonal dynamics, markedly depleting a pre-determined set of chemoresistant clones with increased leukemia-initiating potential, thus leading to chemosensitive relapses. Importantly, we show that these clonal dynamics are not based on genetic differences, but instead on the transcriptional landscape associated with stemness and chemoresistance. We thus provide mechanistic insight for the promise of upfront addition of low-dose DNMTi to standard chemotherapy, to sensitize AML to (re-)treatment; and propose this might circumvent development of chemoresistance in AML relapses, potentially turning this fatal malignancy into a chronic manageable disease.

\begin{abstract}
Methods
Ethics statement. Bone marrow samples of adult AML patients were collected at the Hematology Department at Instituto Português de Oncologia (IPO, Lisbon, Portugal) after written and informed consent and ethical Review Board approval from Instituto Português de Oncologia, in accordance with the Declaration of Helsinki. Samples used in this study were selected for high frequency of immunophenotypically leukemic blasts (over 80\%). All animal experiments were conducted in accordance with standard institutional animal care procedures and followed ethical committee protocols at Instituto de Medicina Molecular João Lobo Antunes.
\end{abstract}

Barcode construction and lentivial-barcode production. The DNA Barcode library containing $\sim 2600$ unique barcodes was produced as previously described ${ }^{68}$.
To produce barcode containing viral particles HEK 293 T (ATCC ${ }^{\circledast}$ CRL-3216 ${ }^{\mathrm{m}}$ ) cell line was used. Cells were plated at 70.000 cells $/ \mathrm{cm}^{2}$ in tissue culture flask in IMDM complete medium composed by Iscove's Modified Dulbecco's Medium GlutaMAX $^{\text {No }}$ Supplement $\left(\right.$ Gibco $^{\oplus}, \mathrm{NY}$, USA) supplemented [10\% (v/v) fetal bovine serum (Gibco $\left.{ }^{\oplus}, \mathrm{NY}, \mathrm{USA}\right), 1 \%(\mathrm{v} / \mathrm{v})$ Sodium Pyruvate (Gibco $\left.{ }^{\oplus}, \mathrm{NY}, \mathrm{USA}\right), 1 \%(\mathrm{v} / \mathrm{v})$ penincillin-streptomycin $\left(\mathrm{Gibco}^{\circledR}, \mathrm{NY}, \mathrm{USA}\right)$ and $0.1 \%(\mathrm{v} / \mathrm{v})$ Gentamycin $\left(\mathrm{Gibco}^{\circledR}\right.$, NY, USA)] during $16-24 \mathrm{~h}$ before transfection, at $37^{\circ} \mathrm{C}$ and $5 \% \mathrm{CO}_{2}$. For cells transfection $0.0272 \mu \mathrm{g} / \mathrm{cm}^{2}$ of Barcode library expression vector, $0.0181 \mu \mathrm{g} / \mathrm{cm}^{2}$ of gag-pol expressing vector and $0.0181 \mu \mathrm{g} / \mathrm{cm}^{2}$ of VSVG expressing vector were mixed with $6.53 \mu \mathrm{L} / \mathrm{cm}^{2}$ serum free medium and $1.11 \mu \mathrm{L} / \mathrm{cm}^{2}$ Lipofectamine 2000 (InvitrogenTM, NY, USA) and incubated for $20 \mathrm{~min}$ at room temperature (RT). Subsequently the medium was removed from the cells and the mixture was immediately added at $14.2 \mu \mathrm{g} / \mathrm{cm}^{2}$ drop-wise and the cultures incubated at $37^{\circ} \mathrm{C}$ and $5 \% \mathrm{CO}_{2}$ for $24 \mathrm{~h}$. Old medium was removed and $0.2 \mathrm{~mL} / \mathrm{cm}^{2}$ of IMDM complete medium supplemented with $10 \mathrm{mM}$ Sodium Butyrate (Sigma ${ }^{\circledR}$, Darmstadt, Germany) was added to the cells and incubated at $37^{\circ} \mathrm{C}$ and $5 \% \mathrm{CO}_{2}$ for $8 \mathrm{~h}$. Cells were washed $2-3 \mathrm{x}$ and $0.133 \mathrm{~mL}$ of complete medium $/ \mathrm{cm}^{2}$ was added. After $24 \mathrm{~h}$ of incubation, medium contained viral particles was harvested and filtrated with $0.45 \mathrm{~mm}$ filter, aliquoted and frozen at $-80^{\circ} \mathrm{C}$.

Barcoding of AML cell lines. Human acute myeloid leukemia cell lines HEL (ATCC, TIB-180" $0^{\mathrm{m}}$ ) and OCI-AML3 (DSMZ, ACC 582) were maintained in Iscove's Modified Dulbecco's Medium GlutaMAX ${ }^{\text {sw }}$ Supplement supplemented with $10 \%$ $(\mathrm{v} / \mathrm{v})$ fetal bovine serum, $1 \%(\mathrm{v} / \mathrm{v})$ Sodium Pyruvate $\left(\mathrm{Gibco}^{\oplus}, \mathrm{NY}, \mathrm{USA}\right), 1 \%(\mathrm{v} / \mathrm{v})$ penincillin-streptomycin and $0.1 \%(\mathrm{v} / \mathrm{v})$ Gentamycin $\left(\mathrm{Gibco}^{\oplus}, \mathrm{NY}, \mathrm{USA}\right)$ at $37^{\circ} \mathrm{C}$ with $5 \% \mathrm{CO}_{2}$. Cells were seeded at $0.2 \times 10^{6}$ cells $/ \mathrm{mL}$ and maintained between $0.2 \times 10^{6}$ cells $/ \mathrm{mL}$ and $0.6 \times 10^{6}$ cells $/ \mathrm{mL}$. To transduce the AML cell lines, $6 \times 10^{6}$ cells were plated per well in a 24 -well plate with IMDM complete medium containing $12 \mu \mathrm{g} / \mu \mathrm{L}$ of polybrene (Merck ${ }^{\oplus}$, Darmstadt, Germany), then the supernatant of viral particles previously obtained was also supplemented with $12 \mu \mathrm{g} / \mu \mathrm{l}$ of polybrene and added to the cells. Control wells without viral supernatant were prepared in parallel. The plates were centrifuged at $1500 \mathrm{~g}$ for $90 \mathrm{~min}$ at $32^{\circ} \mathrm{C}$. Finally, the supernatant was removed, the cells were washed twice by changing the medium and centrifugation at $350 \mathrm{~g}$ for $7 \mathrm{~min}$. The cells were incubated at $37^{\circ} \mathrm{C}$ and $5 \% \mathrm{CO}_{2}$ for $20 \mathrm{~h}$. Initially, serial dilutions of the supernatant were tested, starting in a 1:2 dilution in order to determine the amount of virus that will transduced a maximum of $5 \%$ of the cells in the first $24 \mathrm{~h}$. The $<5 \%$ level of transduction was previously determined to be the level at which $>1$ barcodes are very rarely integrated per cell. In detail, we preformed double transductions using GFP + and RFP + coding viral vectors and determined that double transductions (as measured by dual positivity) were only observed above $~ 10 \%$ levels of cotransduction. After $20 \mathrm{~h}$ incubation transduced cells were collected, washed, stained with $10 \mu \mathrm{l} / \mathrm{mL}$ of 7AAD (Biolegend, USA) and $2.5 \mu \mathrm{L} / \mathrm{mL}$ of AnnexinV-APC (InvitrogenTM, NY, USA) and sorted in a FACSAriaTM III BD (Biosciences). Viable cells were sorted and seeded at different numbers in independent wells. After cell expansion the barcode number of each independent culture was determined and only sublines with approximately $10 \%$ of the barcodes in the library (210-330 barcodes) were used as T0 samples. This level of library usage was previously determined to prevent that the same barcode is found in 2 independent cells transduced with the same library ${ }^{58}$. Multiple aliquots of T0 samples were stored at $-80^{\circ} \mathrm{C}$ in fetal bovine serum supplemented with $10 \%$ DMSO (Sigma ${ }^{\oplus}$, Darmstadt, Germany) to be used as T0 for all experiments. HEL, HEK $293 \mathrm{~T}$ and OCI-AML3 were purchased from ATCC and DSMZ respectively (cell lines were not tested for mycoplasma, nor authenticated).

Xenotransplantation of barcoded AML cells. NOD-Rag1-/- $\gamma c-/-$ (NRGS, 024099) mice were obtained from the Jackson Laboratories (USA). 8-10-weeks-old male and female NRGS mice were sub-lethally irradiated ( $250 \mathrm{cGy}) 24 \mathrm{~h}$ before the injections and kept with oral antibiotic (Bactrim) diluted in the drinking water during all the experiment. For the xenotransplantation, a previously described model of intra bone marrow (IBM) xenografted human AML cells was used ${ }^{54}$. Briefly AML cells (barcoded HEL and OCI-AML3 cell lines) were washed, resuspended in PBS and injected directly into the right tibia bone marrow 10000 cells/mouse. Mice were housed at IMM Lisboa, monitored daily for signs of disease and weekly for tumour burden levels quantification. Animals exhibiting signs of disease (paralysis, more that $20 \%$ of weight loss or high tumour burden) were killed by $\mathrm{CO}_{2}$ and the bone marrow and spleen were immediately collected for posterior analysis.

In vivo leukemia progression evaluation. Leukemia progression was evaluated by weekly quantification of peripheral blood circulating tumour cells. Blood $(70-100 \mu \mathrm{L})$ was collected from facial vein and stored in tubes containing $10 \mu \mathrm{L}$ of Heparin (500un/mL, B.Braun, Germany). In each blood sample, a defined amount of beads (Coulter CC Size Standard L10) was added for absolute cell quantification and Red Cells Lysis Buffer 1x(RBC Lysis Buffer 10x, Biolegend, USA) was added in multiple rounds until complete erythrocyte clearance. Cells were stained with LIVE/ DEADTM dead cell staining kit (InvitrogenTM, NY, USA) for 15 min at $4{ }^{\circ} \mathrm{C}$. Total GFP + Live/Dead- cells were quantified by Flow cytometry on an LSR FortessaII Cell Analyzer (BD Biosciences). Data was analyzed with FlowJo X 10.0.7 software (TreeStar, USA) and the results shown as the absolute numbers per $\mathrm{mL}$ of blood. 
Animals were assigned randomly to treatment groups upon reaching a minimum level of $100 \mathrm{GFP}+\mathrm{CD} 45+$ viable cells $/ \mathrm{mL}$ of blood (treatment threshold).

In vivo chemotherapy drugs treatment. NRGS mice were IBM xenotransplanted with 10000 cells before treatment assignment. Upon reaching tumor load threshold levels mice were treated with: (1) chemotherapy regimen (Doxo + Cyta): intraperitoneal (i.p.) injection of cytarabine $(100 \mathrm{mg} / \mathrm{kg}$ per day over 5 days-Citaloxan $20 \mathrm{mg} / \mathrm{mL}$ stock) and doxorubicin (3 mg/kg per day over the first 3days-doxorubicin chlorohydrate, $2 \mathrm{mg} / \mathrm{mL}$ stock) as previously described ${ }^{55}$. (2) Chemotherapy combined with decitabine (Doxo + Cyta + DAC): chemotherapy was administred as in 1) and decitabine combination was initiated 2 days after Doxo + Cyta, via i.p. injection of $0.5 \mathrm{mg} / \mathrm{kg}$ DAC (5-Aza-2'-deoxycytidine, $5 \mathrm{mg}$, Sigma) for 5 consecutive days ${ }^{69}$. (3) No treatment group (NT) received i.p. PBS. Disease progression was monitored as described. Mice were sacrificed upon signs of illness and viable GFP + AML cells were bone marrow sorted and stored at $-80^{\circ} \mathrm{C}$, for further ex vivo evaluation.

In vivo leukemia-initiating cells evaluation. NRGS mice were xenotransplanted with serial dilutions (100000,10000, 1000, 100, 10 cells) of barcoded HEL and OCIAML3. Starting populations were in vitro generated NT, Doxorubicin (Doxo), Doxorubicin plus Cytarabine (Doxo + Cyta) or Doxorubicin plus Cytarabine and Decitabine (Doxo + Cyta + DAC) relapsing cells. Mice were sacrificed upon signs of illness or upon experiment termination (10 weeks after xenotransplantation). Viable GFP + AML cells were quantified in the injected tibia or in the pool of the remaining tibia, left and right femurs. Animals with human AML cells frequency over $0.5 \%$ of the total mouse leukocyte population in either injected tibia or pooled bone marrow were scored as positive. Leukemia-initiating cell (L-IC) frequency was determined by Extreme Limiting Dilution Analysis (ELDA) software (http://bioinf.wehi.edu.au/ software/elda/index.html), provided by the Walter and Eliza Hall Institute ${ }^{70}$.

\section{Determination of clones with leukemia-initiating potential. NRGS mice were} xenotransplanted with 10000 barcoded HEL (total of seven mice) or OCI-AML3 (total of six mice) NT cell populations with known barcode (BC) architecture and predicted average number of L-ICs. An estimated number of L-ICs per BC-lineage present in the 10,000 xenotransplanted cell population was determined assuming even distribution of L-ICs by the different BC-lineages. After determining the BC arquitecture of in vivo established NT HEL and OCI-AML3 cell populations a Poisson-probability distribution criteria was defined to establish which BC-lineages were more frequently found in vivo (i.e. established in more mice) than expected. These lineages were defined as high leukemia-initiating cell (HiL-IC) lineages.

In vitro chemotherapy treatment of hAML cell lines. All the in vitro treatment regimens were optimized using the mainstream drugs used in clinical management of AML. The different treatment regimens consisted in using only the anthracycline doxorubicin $(1.8 \mu \mathrm{M}$, doxorubicin chlorohydrate, $2 \mathrm{mg} / \mathrm{mL}$, medac), cytarabine $(6 \mu \mathrm{M}$, Citaloxan, $20 \mathrm{mg} / \mathrm{mL}$, Hospira Portugal Lda) or hypomethylating agent decitabine $\left(0.1 \mu \mathrm{M}, 5\right.$-Aza- ${ }^{\prime}$-deoxycytidine, $5 \mathrm{mg}$, Sigma) and using conjugations of this drugs with or without the hypomethylating agent (namely, doxorubicin plus cytarabine and doxorubicin plus cytarabine combined with decitabine). Non-treated cells were cultured in IMDM with $0.1 \mu \mathrm{M}$ of dimethyl sulfoxide (DMSO), as control. The drugs were always added to the cells at time T0h (beginning of each experiment) and each treatment condition was always performed at least in three biological replicates per experiment. Barcoded HEL cells were counted by Trypan blue exclusion method ( $>75 \%$ of viability was ensured), and 6 million cells were seeded at the concentration of $2 \times 10^{5} \mathrm{cells} / \mathrm{mL}$ in the presence or absence of each treatment regimen and incubated for $72-74 \mathrm{~h}$ at $37^{\circ} \mathrm{C}$, $5 \% \mathrm{CO}_{2}$. After incubation, cells were thoroughly washed and enriched for live cells through Ficoll-Paque (Histopaque ${ }^{\circledast}$-1077, sigma) gradient exclusion of dead cells. The recovered live cells were washed three times and reseeded at the concentration of $2 \times 10^{5}$ cells $/ \mathrm{mL}$ in fresh IMDM complete medium. Culture cells were kept growing at $37^{\circ} \mathrm{C}, 5 \% \mathrm{CO}_{2}$ during approximately 30 days until the number of live cells reached the initially seeded number ( 6 million cells). Every 3 or 4 days $1 \%$ of each cell culture was stained with $10 \mu \mathrm{L} / \mathrm{mL}$ of 7AAD (Biolegend, USA) and $2.5 \mu \mathrm{L} /$ $\mathrm{mL}$ of AnnexinV-APC (InvitrogenTM, NY, USA) and analyzed for the total cell numbers and the live/dead cells by flow cytometry on an LSR FortessaII Cell Analyzer (BD Biosciences). Pellets of live cells $\left(1 \times 10^{4}-1 \times 10^{5}\right)$ were collected and frozen for barcode sequencing at indicated timepoints. Cultures that regrew from previous treatment regimens were (re)treated only with chemotherapy for $72 \mathrm{~h}$.

RNA isolation, cDNA production, and real-time PCR. mRNA was extracted from cell lines using High Pure RNA Isolation kit (Roche). Reverse transcription was performed with random oligonucleotides (Invitrogen) using Moloney murine leukemia virus reverse transcriptase (Promega) for $1 \mathrm{~h}$ at $42^{\circ} \mathrm{C}$. Relative quantification of specific cDNA species to endogenous reference human GAPDH was carried out using SYBR on ABI ViiA7 cycler (Applied Biosystems). The CT for the target gene was subtracted from the $\mathrm{CT}$ for endogenous references, and the relative amount was calculated as $2-\triangle \mathrm{CT}$. Primer sequences were the following: $G A P D H$ forward, CTCCTCTGACTTCAACAGCGACAC, GAPDH reverse,

TGCTGTAGCCAAATTCGTTGTCAT, CDKN1C forward, AGAGATCAGCGCC TGAGAAG, reverse, GGGCTCTTTGGGCTCTAAAC
Analysis of stem cell markers and efflux activity. Untreated cells (NT) and treatment-relapsing cells (doxorubicin, cytarabine, doxorubicin plus cytarabine and doxorubicin puls cytarabine combined with decitabine) from barcoded HEL and OCIAML3 cell lines were analysed. Stem-cell surface markers were evaluated by flow cytometry using anti-hCD34-APC (Biolegend, USA), anti-hCD38-PE (Biolegend, USA) and anti-hCD99-PE (Biolegend, USA) antibodies. ABC transporter activity was analysed using the eFluxx-ID Gold multidrug resistance kit (Enzo Life Sciences) according to the manufacturer's instructions.

Quantification and statistical analysis. All $\mathrm{P}$ values were calculated using oneway ANOVA test with GraphPad Prism software, unless otherwise described in the methods or figure legends. No specific randomization or blinding protocol was used for these analyses. Statistically significant differences are indicated with asterisks in figures with the accompanying $\mathrm{P}$ values in the legend. Error bars in figures indicate SD for the number of replicates, as indicated in the figure legend.

Cytosine DNA methylation quantification. Dry pellets of $0.5-1 \times 10^{5}$ live cells were prepared for every condition tested. Cell pellets were treated with RNaseA for $1 \mathrm{~h}$ at $37^{\circ} \mathrm{C}$ and proteinase $\mathrm{K}$ for over-night. Genomic DNA was isolated using phenol chloroform and resuspended in $0.01 \mathrm{M}$ Tris-HCL (pH8) and subsequently quantified using dsDNA BR Assay Kit (Qubit). $1 \mu \mathrm{g}$ of genomic DNA was used for sample preparation by treating with $5 \mathrm{U}$ DNA Degradase Plus at $37^{\circ} \mathrm{C}$ for $1 \mathrm{~h}$ (ZymoResearch, E2021) to obtain individual nucleosides in a final volume of $25 \mu \mathrm{L}$ and inactivated by adding $175 \mu \mathrm{L}$ of $0.1 \%$ formic acid ${ }^{71}$. DNA-me measurements were performed at the VBCF- Vienna Biocenter Core Facilities.

Barcode PCR amplification, deep sequencing and analysis. Barcode quantification was performed as previously described ${ }^{72}$. In detail, dry pellets of $0.5-1 \times 10^{5}$ live cells were prepared for every condition tested. Pellets were resuspended in $40 \mu \mathrm{L}$ of DirectPCR ${ }^{\circledast}$ lysis buffer (Viagen Biotech, USA) containing $200 \mathrm{mg} / \mathrm{mL}$ of proteinase K. The cells were lysed in a thermocycler at $55^{\circ} \mathrm{C}$ for $1 \mathrm{~h}$ and $90^{\circ} \mathrm{C}$ for $30 \mathrm{~min}$. For the first PCR, $3 \mu \mathrm{L}$ of TopLib $5^{\prime}$-TGCTGCCGTCAACTAGAACA-3 and $3 \mu \mathrm{L}$ of BotLib 5'-GATCTCGAATCAGGC GCTTA-3' primers and $50 \mu \mathrm{L}$ of $2 x$ MyTaqTM red Mix (Bioline, UK) were added to the $40 \mu \mathrm{L}$ pellet of each samples. After mixing and before PCR run, $50 \mu \mathrm{l}$ was transferred to an empty PCR tube, to provide technical replicates. The tubes were placed in a thermocycler initially for $5 \mathrm{~min}$ at $94^{\circ} \mathrm{C}$, then $30 \mathrm{cycles}$ of $58^{\circ} \mathrm{C}$ for $15 \mathrm{~s}, 72^{\circ} \mathrm{C}$ for $15 \mathrm{~s}$ and $94^{\circ} \mathrm{C}$ for $15 \mathrm{~s}$ were performed and finally for $10 \mathrm{~min}$ at $72{ }^{\circ} \mathrm{C}$. For the second PCR different index primers were used for every sample and technical replicate. The index forward primers was designed taking into account a production of a library of 384 different 82-bp primers containing unique 8-bp sequence that differed by at least 2 bases, a P7 annealing region for the Illumina ${ }^{\circledR}$ Sequencing system (Illumina ${ }^{\circledR}$ Sequencing, USA)), and a 16-bp annealing region to the first PCR. For the common reverse primer, a sequence that includes a P5 annealing region for the Illumina ${ }^{\circledR}$ Sequencing system followed by an annealing region for the first PCR product $5^{\prime}$ CAAGCAGAAGACGGCATACGAGATTGCTGCCGTCAACTAGAACA- $3^{\prime}$ was designed. For the PCR mixture, $1 \mu \mathrm{L}$ of the first PCR product, $5 \mu \mathrm{L}$ of index primer $1 \mu \mathrm{L}$ of common primer, $15 \mu \mathrm{L}$ of $2 \mathrm{x}$ MyTaqTM red Mix (Bioline, UK) and $8 \mu \mathrm{L}$ of $\mathrm{H} 2 \mathrm{O}$ were mixed together. PCR amplification was carried out as described above, and the presence of the expected 224-bp product was checked for each sample by $2 \%$ agarose gel electrophoresis. After the two PCR, $4 \mu \mathrm{L}$ of each sample containing different indexes up to 384 that were pooled, run on an E-Gel ${ }^{\oplus}$ Size Select $2 \%$ (InvitrogenTM, NY, USA) to obtain the 224-bp product and sequenced on a HiSeqTM 2000 (Illumina ${ }^{\circledR}$ Sequencing, USA). ASCIDEA Computational Biology Solutions, Barcelona, performed the sequencing. A single-run 50-bp sequencing run was sufficient to read through the index, common annealing region, and the first $15 \mathrm{bp}$ of the barcode required for data analysis. Upon sequencing of the barcodes present in each subculture, we analyzed the resulting raw data with a bioinformatic pipeline previously optimized. Barcode sequences were extracted using XCALIBR program (developed at Netherlands Cancer Institute - Genomics Core Facilty) generating tabulated data into a matrix containing the fraction of reads for each barcode versus the indexes. Data was further processed using a customized script in R (kindly provided by Dr. Leïla Prerie, Institute Curie) using three main steps. First excludes samples where there were insufficient read counts from the deep sequencing (average of the two technical replicates $<1 \times 10^{4}$ ). Samples having passed this step were then normalized to $1 \times 10^{5}$ for each sample. Secondly, as a measure of sufficient recovery for subsequent lineage comparisons, we further excluded samples where the two technical replicates did not pass a Pearson correlation coefficient of 0.8 . After, all reads of barcodes present in only one of either technical replicate of a given sample-an indication that there was a low confidence for inclusion of that barcode-were changed to zero $(0)$ reads for that sample and excluded. After confirming that technical replicates were well represented their average was then taken for further analysis.

Total RNA extraction, sequencing, and analysis. Total RNA was extracted from frozen cells pellets using the High Pure RNA Isolation Kit (Roche, Basileia, Swiss) and quantified using QubitTM RNA HS Assay Kit (InvitrogenTM, NY, USA) on the Qubit ${ }^{\circledR}$ 2.0 Fluorometer (InvitrogenTM, NY, USA). ASCIDEA Computational Biology Solutions, Barcelona, performed the sequencing. One microgram of 
high-purity total RNA (defined as having an RNA integrity number greater than 7.0) was used as input for the Illumina TruSeq RNA Sample Prep Kit, Sets A/B (48Rxn) (Illumina). The gel-free protocol was employed for the TruSeq RNA Sample Prep Kit per the manufacturer's specifications and performed on the Beckman Coulter Biomek FXp robotics platform. The standard RNAfragmentation profile was used as recommended by Illumina $\left(94^{\circ} \mathrm{C}\right.$ for $\left.8 \mathrm{~min}\right)$. The PCR-amplified RNA-seq library products were then quantified using the Fragment Analyzer Standard Sensitivity NGS Fragment Analysis Kit (Advanced Analytical Technologies). The samples were diluted to $10 \mathrm{nM}$ in EB Buffer (Qiagen), denatured, and loaded at $2.75 \mathrm{pM}$ on an Illumina HiSeq2000 in Rapid Run Mode using TruSeq Rapid PE Cluster Kit-HS and TruSeq Rapid SBS Kit-HS (200 cycle) reagents (Illumina). The RNA-seq libraries were sequenced at $100 \mathrm{bp}$ paired-end with a 7-bp index using the standard Illumina primers. Quality of the reads obtained by HiSeq2000 sequencing was checked with FastQC software (http://www.bioinformatics.bbsrc.ac.uk/projects/fastqc/). Preprocessing of the reads was performed with fastx-toolkit (http://hannonlab.cshl.edu/fastx_toolkit/ index.htmL) and aScidea specific perl scripts property of aScidea (http://www. ascidea.com) in order to filter regions of low quality. Adaptors and low quality bases at the ends of sequences and reads with undetermined bases or with $80 \%$ of their bases with less than $20 \%$ quality score were trimmed. Raw reads that passed the quality filter threshold were mapped using Bowtie2 2.2.8 to generate read alignments for each sample. The reference genome used was the Homo Sapiens version GRCh38. The transcript isoform level and gene level counts were calculated using FeatureCounts from SubRead Package. Differential transcript expression was then computed using DESeq2. The resulting lists of differentially expressed isoforms were filtered by $\ln$ (fold_change) $>1$ and $<-1$ and adjusted $p$-value of 0.05 . Gene set enrichment analysis (GSEA) was performed with the GSEA v2.0 software (Broad Institute of MIT (Massachusetts Institute of Technology) and Harvard, http://www.broad.mit.edu/gsea) on pre-ranked lists of differentially expressed genes. Normalized enrichment scores (NES) with $P$ values $<0.05$ and false discovery rates $(\mathrm{FDR})<0.05$ were considered statistically significant. Initial GSEA analysis was performed on HALLMARK gene set terms ${ }^{73}$.

Exome sequencing and analysis. Total DNA was recovered by DNeasy Blood \& Tissue Kit (Qiagen, USA) and quantified using QubitTM dsDNA HS Assay Kit (InvitrogenTM, NY, USA) on the Qubit ${ }^{\circledR}$ 2.0 Fluorometer (InvitrogenTM, NY, USA). Fragmentation of $1 \mu \mathrm{g}$ of genomic DNA was performed using adaptive focused acoustic technology (AFA; Covaris). The fragmented DNA was repaired; an " $\mathrm{A}$ " is ligated to the $3^{\prime}$ end, agilent adapters are then ligated to the fragments. Once ligation was assessed, the adapter-ligated product was PCR amplified. The final purified product was quantified using $\mathrm{QPCR}$ according to the $\mathrm{qPCR}$ Quantification Protocol Guide and qualified using the Caliper LabChipHigh Sensitivity DNA (PerkinElmer). For exome capture, $250 \mathrm{ng}$ of DNA library was mixed with hybridization buffers, blocking mixes, RNase block and $5 \mu \mathrm{L}$ of SureSelect all exon capture library, according to the standard Agilent SureSelect Target Enrichment protocol. Hybridization to the capture baits was conducted at $65^{\circ} \mathrm{C}$ using heated thermal cycler lid option at $105^{\circ} \mathrm{C}$ for $24 \mathrm{~h}$ on PCR machine. The captured DNA was then amplified. The final purified product was then quantified using qPCR according to the qPCR Quantification Protocol Guide, qualified using the TapeStation DNA screentape (Agilent) and then sequenced using the HiSeq 4000 platform (Illumina,San Diego, USA). Mapping and alignment were carried out as follows. First, read files (Fastq) were generated from the sequencing platform using the manufacturer's proprietary software. Mapping and alignment were carried out as follows. First, read files (Fastq) were generated from the sequencing platform using the manufacturer's proprietary software. Reads were mapped to their location in the reference human genome (GRCh38) using the Burrows-Wheeler Aligner (BWA) package, version 0.6.2. Duplicate reads were marked and removed using Samtools rmdup. Freebayes was used to call join variants on all samples and varaint frequencies were then extracted from the vcf file.

Reporting summary. Further information on research design is available in the Nature Research Reporting Summary linked to this article.

\section{Data availability}

RNA-seq data has been deposited in NCBI's Gene Expression Omnibus (GEO) and is accessible using the accession number GSE134506. Whole exome sequencing data has been deposited in NCBI's Sequence Read Archive (SRA) and is accessible via SRA under the accession number PRJNA555070. All the other data supporting the findings of this study are available within the article and its supplementary information files and from the corresponding author upon reasonable request. A reporting summary for this article is available as a Supplementary Information file. The source data underlying Figs. 1-6 and Supplementary Figs. 1-11 are provided as a Source Data file

Received: 14 February 2019; Accepted: 9 October 2019; Published online: 01 November 2019

\section{References}

1. Holohan, C., Van Schaeybroeck, S., Longley, D. B. \& Johnston, P. G. Cancer drug resistance: an evolving paradigm. Nat. Rev. Cancer 13, 714-726 (2013).

2. Caiado, F., Silva-Santos, B. \& Norell, H. Intra-tumour heterogeneity - going beyond genetics. FEBS J. 283, 2245-2258 (2016).

3. Aparicio, S. \& Caldas, C. The implications of clonal genome evolution for cancer medicine. N. Engl. J. Med. 368, 842-851 (2013).

4. Almendro, V., Marusyk, A. \& Polyak, K. Cellular heterogeneity and molecular evolution in cancer. Annu. Rev. Pathol. Mech. Dis. 8, 277-302 (2013).

5. Batlle, E. \& Clevers, H. Cancer stem cells revisited. Nat. Med 23, 1124-1134 (2017).

6. Ferrara, F. \& Schiffer, C. A. Acute myeloid leukaemia in adults. Lancet (Lond., Engl.) 381, 484-495 (2013).

7. Ding, L. et al. Clonal evolution in relapsed acute myeloid leukaemia revealed by whole-genome sequencing. Nature 481, 506-510 (2012).

8. Garg, M. et al. Profiling of somatic mutations in acute myeloid leukemia with FLT3-ITD at diagnosis and relapse. Blood 126, 2491-2501 (2015).

9. Kronke, J. et al. Clonal evolution in relapsed NPM1-mutated acute myeloid leukemia. Blood 122, 100-108 (2013).

10. Parkin, B. et al. Clonal evolution and devolution after chemotherapy in adult acute myelogenous leukemia. Blood 121, 369-377 (2013).

11. Hackl, H., Astanina, K. \& Wieser, R. Molecular and genetic alterations associated with therapy resistance and relapse of acute myeloid leukemia. $J$. Hematol. Oncol. 10, 51 (2017).

12. Welch, J. S. et al. The origin and evolution of mutations in acute myeloid leukemia. Cell 150, 264-278 (2012).

13. Papaemmanuil, E. et al. Genomic classification and prognosis in acute myeloid leukemia. N. Engl. J. Med. 374, 2209-2221 (2016).

14. Mardis, E. R. et al. Recurring mutations found by sequencing an acute myeloid leukemia genome. N. Engl. J. Med. 361, 1058-1066 (2009).

15. Network, T. C. G. A. R. Genomic and epigenomic landscapes of adult de novo acute myeloid leukemia. N. Engl. J. Med. 368, 2059-2074 (2013).

16. Alexandrov, L. B. et al. Signatures of mutational processes in human cancer. Nature 500, 415-421 (2013).

17. Eppert, K. et al. Stem cell gene expression programs influence clinical outcome in human leukemia. Nat. Med 17, 1086-1093 (2011).

18. Ng, S. W. K. et al. A 17-gene stemness score for rapid determination of risk in acute leukaemia. Nature 540, 433-437 (2016).

19. Shlush, L. I. et al. Tracing the origins of relapse in acute myeloid leukaemia to stem cells. Nature 547, 104-108 (2017).

20. Figueroa, M. E. et al. DNA methylation signatures identify biologically distinct subtypes in acute myeloid leukemia. Cancer Cell 17, 13-27 (2010).

21. Marcucci, G. et al. Epigenetics meets genetics in acute myeloid leukemia: clinical impact of a novel seven-gene score. J. Clin. Oncol. 32, 548-556 (2014).

22. $\mathrm{Li}, \mathrm{S}$. et al. Distinct evolution and dynamics of epigenetic and genetic heterogeneity in acute myeloid leukemia. Nat. Med 22, 792-799 (2016).

23. Luskin, M. R. et al. A clinical measure of DNA methylation predicts outcome in de novo acute myeloid leukemia. JCI insight 1, e87323 (2016).

24. Al-Ali, H. K., Jaekel, N. \& Niederwieser, D. The role of hypomethylating agents in the treatment of elderly patients with AML. J. Geriatr. Oncol. 5, 89-105 (2014).

25. Song, L. X. et al. Clinical outcome of treatment with a combined regimen of decitabine and aclacinomycin/cytarabine for patients with refractory acute myeloid leukemia. Ann. Hematol. 91, 1879-1886 (2012).

26. Jiang, $\mathrm{X}$. et al. The hypomethylating agent decitabine prior to chemotherapy improves the therapy efficacy in refractory/relapsed acute myeloid leukemia patients. Oncotarget 6, 33612-33622 (2015)

27. Li, L. et al. Low-dose hypomethylating agent decitabine in combination with aclacinomycin and cytarabine achieves a better outcome than standard FLAG chemotherapy in refractory/relapsed acute myeloid leukemia patients with poorrisk cytogenetics and mutations. Onco. Targets Ther. 11, 6863-6870 (2018).

28. Bhang, H. C. et al. Studying clonal dynamics in response to cancer therapy using high-complexity barcoding. Nat. Med 21, 440-448 (2015).

29. Naik, S. H., Schumacher, T. N. \& Perié, L. Cellular barcoding: a technical appraisal. Exp. Hematol. 42, 598-608 (2014).

30. Öz, S. et al. Quantitative determination of decitabine incorporation into DNA and its effect on mutation rates in human cancer cells. Nucleic Acids Res. 42, e152-e152 (2014).

31. Zhao, W., Du, Y., Ho, W. T., Fu, X. \& Zhao, Z. J. JAK2V617F and p53 mutations coexist in erythroleukemia and megakaryoblastic leukemic cell lines. Exp. Hematol. Oncol. 1, 15 (2012).

32. Saxton, R. A. \& Sabatini, D. M. mTOR signaling in growth, metabolism, and disease. Cell 168, 960-976 (2017)

33. Drolle, $\mathrm{H}$. et al. Hypoxia regulates proliferation of acute myeloid leukemia and sensitivity against chemotherapy. Leuk. Res. 39, 779-785 (2015).

34. Schillert, A., Trumpp, A. \& Sprick, M. R. Label retaining cells in cancer - The dormant root of evil? Cancer Lett. 341, 73-79 (2013).

35. Zou, P. et al. p57Kip2 and p27Kip1 cooperate to maintain hematopoietic stem cell quiescence through interactions with Hsc70. Cell Stem Cell 9, 247-261 (2011). 
36. Matsumoto, A. et al. p57 is required for quiescence and maintenance of adult hematopoietic stem cells. Cell Stem Cell 9, 262-271 (2011).

37. Kentsis, A. et al. Autocrine activation of the MET receptor tyrosine kinase in acute myeloid leukemia. Nat. Med 18, 1118-1122 (2012).

38. Zhang, J., Gu, Y. \& Chen, B. Mechanisms of drug resistance in acute myeloid leukemia. OncoTargets Ther. 12, 1937-1945 (2019).

39. Marzac, C. et al. ATP binding cassette transporters associated with chemoresistance: transcriptional profiling in extreme cohorts and their prognostic impact in a cohort of 281 acute myeloid leukemia patients. Haematologica 96, 1293-1301 (2011).

40. Behbehani, G. K. et al. Mass cytometric functional profiling of acute myeloid leukemia defines cell-cycle and immunophenotypic properties that correlate with known responses to therapy. Cancer Disco. 5, 988-1003 (2015).

41. Ebinger, S. et al. Characterization of rare, dormant, and therapy-resistant cells in acute lymphoblastic leukemia. Cancer Cell 30, 849-862 (2016).

42. Lapidot, T. et al. A cell initiating human acute myeloid leukaemia after transplantation into SCID mice. Nature 367, 645-648 (1994).

43. Bonnet, D. \& Dick, J. E. Human acute myeloid leukemia is organized as a hierarchy that originates from a primitive hematopoietic cell. Nat. Med 3, 730-737 (1997).

44. Hope, K. J., Jin, L. \& Dick, J. E. Acute myeloid leukemia originates from a hierarchy of leukemic stem cell classes that differ in self-renewal capacity. Nat. Immunol. 5, 738-743 (2004).

45. Ho, T.-C. et al. Evolution of acute myelogenous leukemia stem cell properties after treatment and progression. Blood 128, 1671-1678 (2016).

46. van Rhenen, A. et al. High stem cell frequency in acute myeloid leukemia at diagnosis predicts high minimal residual disease and poor survival. Clin. Cancer Res. 11, 6520-6527 (2005).

47. Zeijlemaker, W. et al. CD34+CD38- leukemic stem cell frequency to predict outcome in acute myeloid leukemia. Leukemia. https://doi.org/10.1038/ s41375-018-0326-3 (2018)

48. Wong, D. J. et al. Module map of stem cell genes guides creation of epithelial cancer stem cells. Cell Stem Cell 2, 333-344 (2008).

49. Ivanova, N. B. et al. A stem cell molecular signature. Science 298, 601-604 (2002).

50. Boquest, A. C. Isolation and transcription profiling of purified uncultured human stromal stem cells: alteration of gene expression after in vitro cell culture. Mol. Biol. Cell 16, 1131-1141 (2005).

51. Yamashita, T. et al. EpCAM-positive hepatocellular carcinoma cells are tumor-initiating cells with stem/progenitor cell features. Gastroenterology 136, 1012-1024 (2009)

52. Gal, H. et al. Gene expression profiles of AML derived stem cells; similarity to hematopoietic stem cells. Leukemia 20, 2147-2154 (2006).

53. Kreso, A. \& Dick, J. E. Evolution of the cancer stem cell model. Cell Stem Cell 14, 275-291 (2014).

54. Nóbrega-Pereira, S. et al. VEGFR2-mediated reprogramming of mitochondrial metabolism regulates the sensitivity of acute myeloid leukemia to chemotherapy. Cancer Res. 78, 731-741 (2018).

55. Zuber, J. et al. Mouse models of human AML accurately predict chemotherapy response. Genes Dev. 23, 877-889 (2009).

56. Barve, A. et al. Comparative utility of NRG and NRGS mice for the study of normal hematopoiesis, leukemogenesis, and therapeutic response. Exp. Hematol. https://doi.org/10.1016/j.exphem.2018.08.004 (2018)

57. Oronsky, B. et al. Rewriting the epigenetic code for tumor resensitization: a review. Transl. Oncol. 7, 626-631 (2014).

58. Thomas, D. \& Majeti, R. Biology and relevance of human acute myeloid leukemia stem cells. Blood 129, 1577-1585 (2017).

59. Radujkovic, A. et al. Expression of CDKN1C in the bone marrow of patients with myelodysplastic syndrome and secondary acute myeloid leukemia is associated with poor survival after conventional chemotherapy. Int. J. Cancer 139, 1402-1413 (2016).

60. Steinbach, D. \& Legrand, O. ABC transporters and drug resistance in leukemia: Was P-gp nothing but the first head of the Hydra? Leukemia 21, 1172-1176 (2007).

61. Bröske, A.-M. et al. DNA methylation protects hematopoietic stem cell multipotency from myeloerythroid restriction. Nat. Genet. 41, 1207-1215 (2009).

62. Trowbridge, J. J. et al. Haploinsufficiency of Dnmt1 impairs leukemia stem cell function through derepression of bivalent chromatin domains. Genes Dev. 26, 344-349 (2012).

63. Tsai, H.-C. et al. Transient low doses of DNA-demethylating agents exert durable antitumor effects on hematological and epithelial tumor cells. Cancer Cell 21, 430-446 (2012).

64. Zhang, J. \& Babic, A. Regulation of the MET oncogene: molecular mechanisms. Carcinogenesis 37, 345-355 (2015).

65. Ghobrial, I. M., Detappe, A., Anderson, K. C. \& Steensma, D. P. The bonemarrow niche in MDS and MGUS: implications for AML and MM. Nat. Rev. Clin. Oncol. 15, 219-233 (2018).

66. Cassidy, J. W., Caldas, C. \& Bruna, A. Maintaining tumor heterogeneity in patient-derived tumor xenografts. Cancer Res. 75, 2963-2968 (2015).
67. Shaffer, S. M. et al. Rare cell variability and drug-induced reprogramming as a mode of cancer drug resistance. Nature 546, 431-435 (2017).

68. Schepers, K. et al. Dissecting T cell lineage relationships by cellular barcoding. J. Exp. Med. 205, 2309-2318 (2008).

69. Leonard, S. M., Perry, T., Woodman, C. B. \& Kearns, P. Sequential treatment with cytarabine and decitabine has an increased anti-leukemia effect compared to cytarabine alone in xenograft models of childhood acute myeloid leukemia. PLoS One 9, e87475 (2014).

70. Hu, Y. \& Smyth, G. K. ELDA: extreme limiting dilution analysis for comparing depleted and enriched populations in stem cell and other assays. $J$. Immunol. Methods 347, 70-78 (2009).

71. Capuano, F., Mülleder, M., Kok, R., Blom, H. J. \& Ralser, M. Cytosine DNA methylation is found in Drosophila melanogaster but absent in Saccharomyces cerevisiae, Schizosaccharomyces pombe, and other yeast species. Anal. Chem. 86, 3697-3702 (2014).

72. Naik, S. H. et al. Diverse and heritable lineage imprinting of early haematopoietic progenitors. Nature 496, 229-232 (2013).

73. Liberzon, A. et al. The molecular signatures database (MSigDB) hallmark gene set collection. Cell Syst. 1, 417-425 (2015).

\section{Acknowledgements}

We thank Leila Perie (Institut Curie, Paris), Shalin Naik (Walter and Eliza Hall Institute, Melbourne) Nuno Barbosa-Morais (iMM-JLA), Stefan Butz (DMMD, Zurich), Massimiliano Manzo (DMMD, Zurich) and Daniel Sobral (UCIBIO, Portugal) for technical advice; Inês Pereira, MD (Instituto Português de Oncologia-Francisco Gentil, Lisbon) for patient sample collection and the Animal, Bioimaging, Flow Cytometry facilities and the other members of the Silva-Santos lab (iMM-JLA) for support. This work was funded by the European Research Council (CoG_646701 to B.S-S. and AdG Life-His-T to T.N.S.) and Fundação para a Ciência e Tecnologia (SFRH/BPD/91344/2012 to F.C.; Welcome II programme, SFRH/BCC/105888/2014, SFRH/BPD/112968/2015 and EXPL/BIM/ONC/ 1656/2013 to H.N.; and UID/BIM/50005/2019 project funded by Fundação para a Ciência e a Tecnologia (FCT)/ Ministério da Ciência, Tecnologia e Ensino Superior (MCTES) through Fundos do Orçamento de Estado.

\section{Author contributions}

F.C., H.N. and B.S-S. designed research and wrote the paper; F.C., H.N., D.M-S., C.J., N.S, T.C., C.R., R.F., B.K. and A.E.S. performed experiments and analyzed the data; T.B., C.R.V., M.G.S., M.G.M., and T.N.S provided key research tools; B.S-S. and H.N. supervised research.

\section{Competing interests}

The authors declare no competing interests.

\section{Additional information}

Supplementary information is available for this paper at https://doi.org/10.1038/s41467019-12983-z.

Correspondence and requests for materials should be addressed to F.C., H.N. or B.S.-S.

Peer review information Nature Communications thanks Leonid Bystrykh and the other, anonymous, reviewer(s) for their contribution to the peer review of this work. Peer reviewer reports are available.

Reprints and permission information is available at http://www.nature.com/reprints

Publisher's note Springer Nature remains neutral with regard to jurisdictional claims in published maps and institutional affiliations.

Open Access This article is licensed under a Creative Commons Attribution 4.0 International License, which permits use, sharing, adaptation, distribution and reproduction in any medium or format, as long as you give appropriate credit to the original author(s) and the source, provide a link to the Creative Commons license, and indicate if changes were made. The images or other third party material in this article are included in the article's Creative Commons license, unless indicated otherwise in a credit line to the material. If material is not included in the article's Creative Commons license and your intended use is not permitted by statutory regulation or exceeds the permitted use, you will need to obtain permission directly from the copyright holder. To view a copy of this license, visit http://creativecommons.org/ licenses/by/4.0/

(c) The Author(s) 2019 